\title{
Additional Experimental Confirmations of the New Chemical Species of Santilli Magnecules
}

\author{
Y. Yang ${ }^{1}$, J. V. Kadeisvili ${ }^{1,2, *}$ and S. Marton ${ }^{1}$ \\ ${ }^{1}$ MagneGas Corporation, 150 Rainville Rd, Tarpon Springs, FL, 34689, U.S.A.; ${ }^{2}$ Institute for Basic Research, 35246 US \\ 19 N, No. 215. Palm Harbor, FL 34684, U.S.A.
}

\begin{abstract}
In this paper, we present experimental confirmations of the new chemical species of magnecules identified by R. M. Santilli in 1998 [1], with particular reference to: stability of magnecules at ambient temperature; progressive reduction of magnecular species with the increase of the temperature; termination of magnecular species at a suitable Curie temperature; presence in the magnecular clusters of weakly bonded individual atoms, dimers, and conventional molecules; detection of magnecular clusters under suitable mass spectroscopy; transparency to infrared detectors at the a.m.u. of the clusters (and not at smaller a.m.u. characterizing constituents); dependence of the detected clusters from the elusion time; anomalous adhesion of magnecules to various substances; anomalous mutation of magnecular clusters; anomalous accretion of magnecules by one Hydrogen atom; and other features. It is stressed that conventional gas chromatographic equipment and procedures (that are so effective for the detection of molecules) are ineffective, if not misleading, for the detection of the new species of magnecules, thus explaining the protracted lack of general detection of mag-necules and the need for specialized equipment and procedures. The paper ends with the indication of the environmental advantages of fuels with Santillimagnecular structure due to the general lack of contaminants in the exhaust. The authors shall make available samples of magnecular gases at no cost to qualified chemists for independent analyses.
\end{abstract}

Keywords: Valence, molecules, magnecules, magnecular bond, magnecular fuels.

\section{INTRODUCTION}

In memoir [1] of 1998, the Italian-American physicist R.

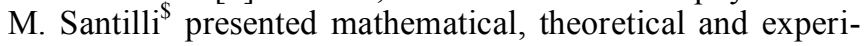
mental evidence on the existence of the new chemical species of magnecules defined as clusters of individual atoms $(\mathrm{H}, \mathrm{O}, \mathrm{C}$, etc.), dimers $(\mathrm{HO}, \mathrm{CH}$, etc.) and ordinary molecules $(\mathrm{H} 2, \mathrm{CO}, \mathrm{H} 2 \mathrm{O}$, etc.) bonded together by attractive forces between opposing magnetic polarities of toroidal polarizations of atomic orbitals, as well as the polarization of the magnetic moments of nuclei and electrons (see a conceptual rendering in Fig. 1).

Santilli suggested the name "magnecules" in order to distinguish the new species from conventional "molecules" (namely stable clusters of atoms under the conventional valence bond), as well as to indicate the primary magnetic origin of the new bond. The symbol "- " is widely used to denote a valence bond (such as $\mathrm{H}-\mathrm{H}$ ) while the symbol "x" is used to denote a magnecular bond (such as $\mathrm{H} \mathrm{x} \mathrm{H}$ ).

The main theoretical argument of Ref. [1] is that the toroidal polarization of the electron orbitals creates a magnetic field (due to the rotation of the electrons within said toroid) which does not exist for the same atom when the electron orbitals have the conventional spherical distribution. When two so polarized atoms are at a sufficiently close distance,

*Address correspondence to this author at the MagneGas Corporation, 150 Rainville Rd, Tarpon Springs, FL, 34689, U.S.A.; Tel: +1-727 -6883992; Fax: +1-727 -9346260; Email: research@magnegas.com

${ }^{\$}$ See the CV: http://www.world-lecture-series.org/santilli-cv PACS: 05.70.Ln, 07.75.+h, 32.10.Bi the resulting total force between the two atoms is attractive because all acting forces are attractive except for the repulsive forces due to nuclear and electron charges. However, the latter forces can be averaged to zero in first approximation since the individual atoms have a null total charge. Alternatively, individual atoms can be assumed in first approximation to have a null total charge distribution, resulting in the evident dominance of the attractive magnetic forces between two atoms with toroidal polarization of their orbitals.

Memoir [1] then provides means for the actual creation of a gas with the new magnecular chemical structure. In essence, Santilli recalled the well-known property that the polarization of the electron orbitals from their natural spherical distribution to the needed toroidal form requires extremely high magnetic fields (expected to be of the order of 1010 Gauss or more) that, as such, are not available in our macroscopic environment.

Therefore, for the creation of the new magnecular species, Santilli suggested the use of a DC electric arc between graphite electrodes submerged within a liquid (such as distilled water). As it is well known, the arc decomposes the liquid molecules into mostly ionized atoms by creating between the tip of the electrodes plasma composed of $\mathrm{H}, \mathrm{C}$, and $\mathrm{O}$ individual atoms, $\mathrm{CH}$ and $\mathrm{OH}$ dimers, and ordinary molecules such as $\mathrm{CO}, \mathrm{H} 2 \mathrm{O}$ and others. Santilli then noted that at atomic distance from said electric arc, the magnetic field has indeed the desired strength since said magnetic field is inversely proportional to the distance (of the order of $10-8 \mathrm{~cm}$ ) and directly proportional to the electric current (of the order of $103 \mathrm{~A}$ or more), thus having a strength of the 
order of 10 nGauss which is sufficient to achieve the desired toroidal polarizations of the electron orbitals (see the conceptual rendering of Fig. 2).

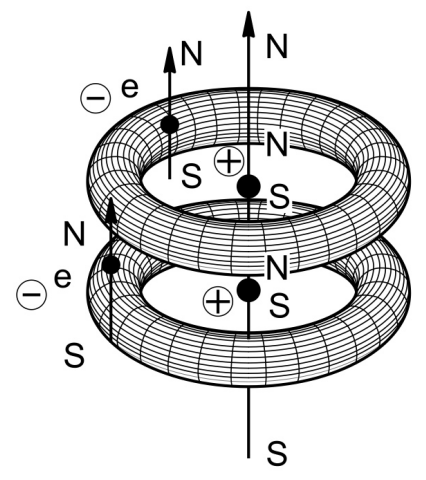

Fig. (1). Conceptual rendering of Santillimagnecules, here referred to the species $\mathrm{H} \times \mathrm{H}$ assumed at absolute zero degree temperature. Note that: the toroidal polarization of the electron orbitals creates a new magnetic fieJd not existing for spherical distributions; all magnetic forces between said toroids as well as between the magnetic polarities of nuclei and electrons are attractive; and the repulsive forces between nuclear and electron charges can be averaged to zero in first approximation since the atoms are individually neutral, thus resulting in the dominance of a new non-valence bond Santilli calls "magnecular." Note that the new bond can be also called "axial" (or "perpendicular") in the sense that the magnetic force exists along the symmetry axis of the toroid (or the magnetic force is perpendicular to the symmetry plane of the toroid).

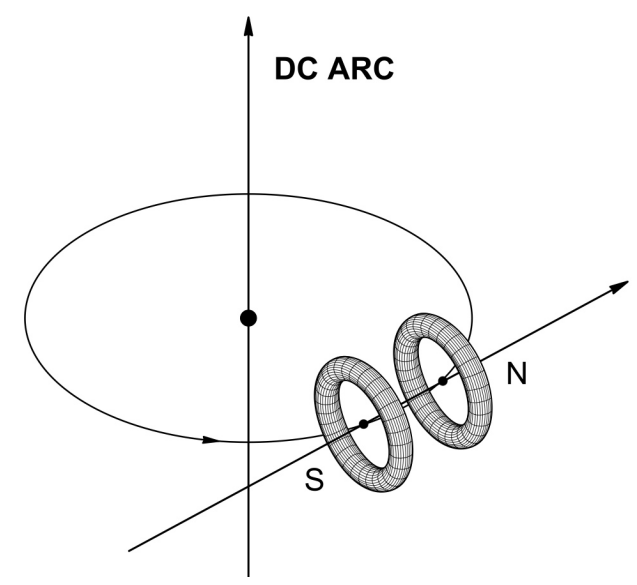

Fig. (2). Conceptual rendering of Santilli's production of a gas with magnecular structure via the use of a submerged electric arc that, at atomic distance, is sufficiently powerful to polarize electron orbitals into toroids and then align polarized atoms essentially along the configuration of (Fig. 1).

Additionally, the strong magnetic field surrounding a DC arc naturally aligns polarized atoms in the needed sequence of magnetic polarities South-North-South-North, etc. resulting in the configuration of (Fig. 1). It is evident that, as soon as the arc is disconnected, atoms return to their natural spherical distribution due to collisions and other reasons. Santilli's main chemical argument is that the spherical distribution is indeed recovered but for the bonded pairs of polarized atoms as in (Fig. 1), since said spherical distribution cannot be achieved for each individual atoms of the bonded pair due to insufficient energies to break said bond.

Memoir [1] then presented a considerable experimental evidence on the existence of the new species of magnecules for a gas created via the above reviewed method. The verification (reviewed in more details in Section 2) was achieved via the use of a GC-MS/IRD, namely, a Gas Chromatographer Mass Spectrometer equipped with an InfraRed Detector. Santilli's main experimental argument is that all gas chromatographic equipment available in the late 1990s had been conceived and established for the detection of conventional molecular species.

The only possibility available at the time of memoir [1] to ascertain the existence of the new species of magnecules was that of subjecting the same injection, first for detection via the GC-MS and then detection via the IRD. The identification of clear clusters in the GC-MS that have no IR signature establishes the existence of magnecules since their bond is stable at ambient temperature, but weaker than the valence bond, thus having no IR signature. The magnecular nature of the bond was then confirmed by the arc method for its creation. Santilli insisted that it was impossible to achieve the same results in a resolutory way via two separate instruments due to the impossibility of matching scans in the GC-MS with scans in a separate IRD without any ambiguities.

Gas chromatographic analyses reported in memoir [1] were conducted via a GC-MS/IRD consisting of a HP GC model 5890, a HP MS model 5972, and a HP IRD model 5965 operated in rather unusual conditions described in details in ref. [1], such as: largest available feeding line of at least $0.3 \mathrm{~mm}$ ID; cryogenic cooling of the feeding line; lowest available column temperature of $100 \mathrm{C}$; longest available elusion time of about $25 \mathrm{~m}$; and other conditions. Representative chromatographs out of a considerable number of scans of memoir [1] are reported in (Figs. 3-5). Memoir [1] also reported the confirmation of the results (here not reproduced for brevity) obtained via an identical GC-MS/IRD located at a different laboratory.

Memoir [1] also provided considerable experimental evidence for the existence of magnecules in liquids, and comments on the expected existence of magnecular bonds in solids.

Following the appearance of memoir [1], M.G. Kucherenko and A.K. Aringazin [2] confirmed in Ref. [2] of 1998 the numerical value computed by Santilli for the magnetic field along the axial symmetry of a toroidal polarization of the electron orbital of the Hydrogen atom as being about 1, 347 times stronger than the nuclear magnetic field, thus confirming the presence of a magnetic field sufficiently strong to create a bond of the type of (Fig. 1).

A. K. Aringazin conducted in Ref. [3] of 2001 in depth theoretical analysis of the strong magnetic fields needed to achieve the toroidal polarization of electron orbitals, thus confirming Santilli's use of a submerged DC electric arc in order to achieve a magnetic field of the needed strength.

In 2001, Santilli released monograph [4] on a systematic presentation of the status of the knowledge in the new field in early 2000. In particular, magnecules were presented in their proper technical environment, that of a nonunitary 

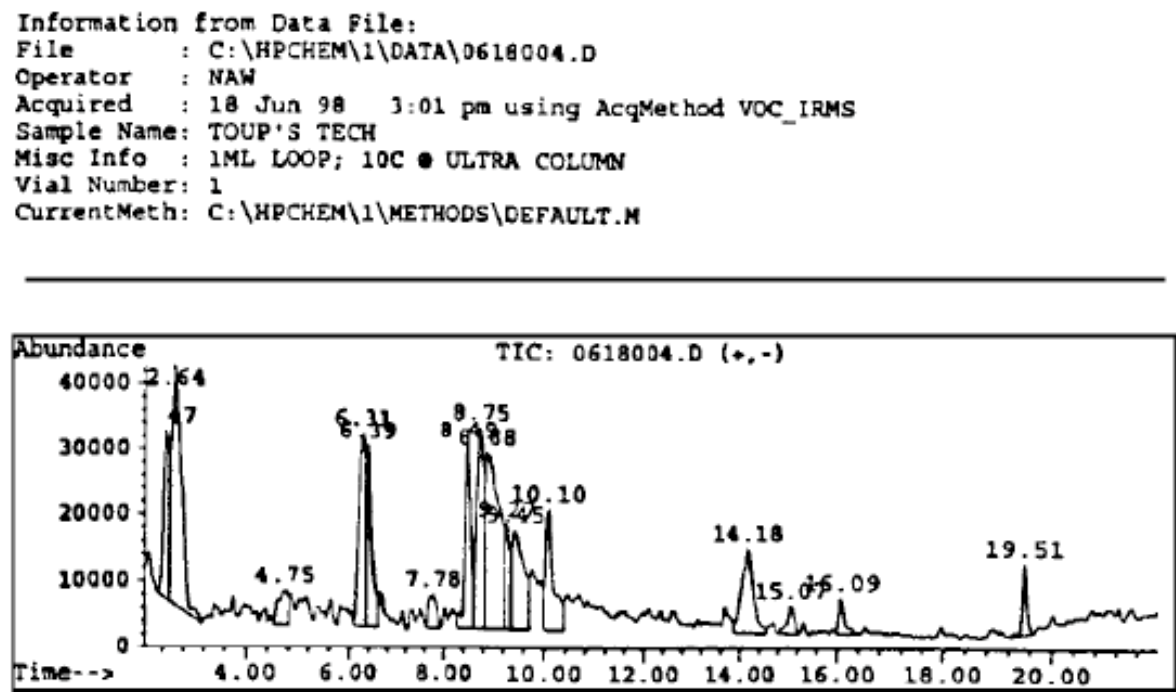

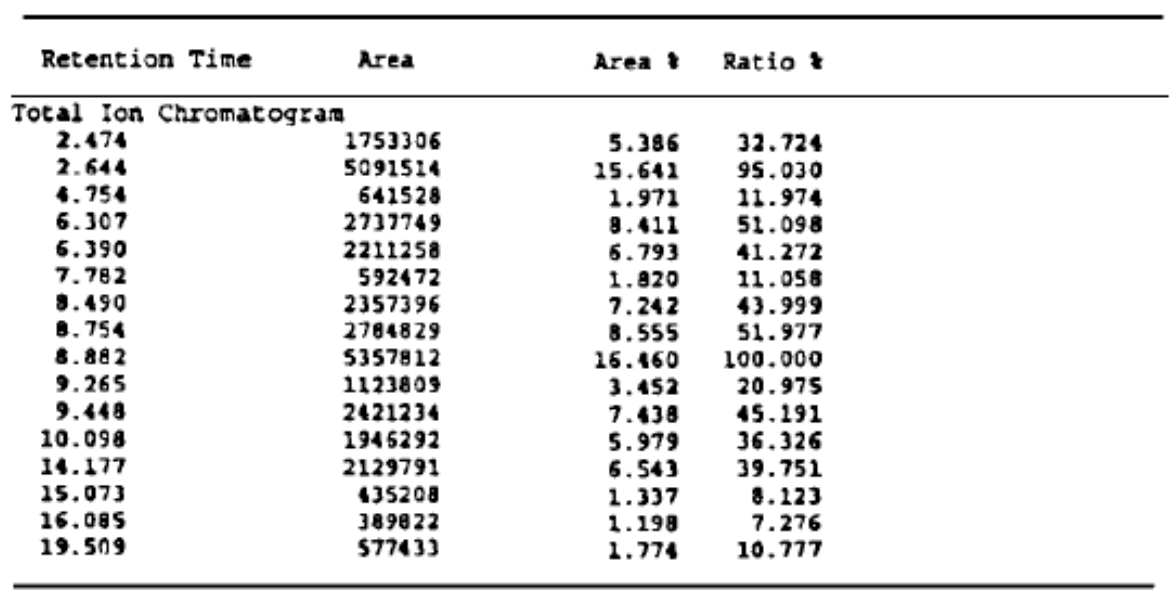

Fig. (3). A reproduction from memoir [1] of the GC-MS peaks between 40 and 400 a.m.u. on a gas with a magnecular structure obtained on June 19, 1998, by analysts Louis A. Dee and Norman Wade of the branch of National Technical Systems (NTS) located at the McClellan Air Force Base in North Highland, near Sacramento, California. The test was done on a type of magnegas produced via an electric arc between graphite electrodes submerged in tap water. Therefore, only the $\mathrm{CO} 2$ peak was expected to appear in the scan in a macroscopic percentage.

covering of quantum chemistry nowadays known under the name of isotopic branch of hadronic chemistry. Monograph [4] also detailed the first industrial realization of the the new species of magnecules consisting of the gaseous fuel produced and sold world wide under the trade name of MagneGasTM (MG) by the U. S. publicly traded company Magnegas Corporation (see www.magnegas.com for details). This application essentially consists of the gasification of liquids via a submerged electric arc into the gaseous fuel MagneGas that possesses anomalous features, such as a combustion flame temperate double that of natural gas, metal cutting faster than acetylene, combustion exhaust without hydrocarbons thanks to a full combustion (because the magnecular bond is weaker than the valence bond), and other features.

Santilli presented in Ref. [5] of 2003 theoretical and experimental evidence for the second industrial application of the new species of magnecules, consisting of the synthesis of "heavy Hydrogen and Oxygen," nowadays denoted with the chemical symbols $\mathrm{MH}$ and MO, respectively, and consisting of magnecular clusters of Hydrogen or Oxygen atoms, re- spectively. When detected via GC-TCD equipment operated at high temperature (so as to destroy the magnecular bond), $\mathrm{MH}$ and $\mathrm{MO}$ can be constituted by conventional Hydrogen or Oxygen up to $99 \%$ pure, yet their specific weight is a multiple that of conventional molecular Hydrogen and Oxygen.

The new species MH and MO were presented in Ref. [5] as evidence on the very existence of magnecules since the Hydrogen has only one valence electron to share and, as such, cannot possibly achieve valence bonds for more than two Hydrogen atoms (see the forthcoming paper [16] for details).

Santilli then provided in Ref. [6] of 2006 theoretical experimental evidence on a third industrial application of the new species of magnecules, here referred to the gas commercially produced via certain electrolyzers and essentially consisting of 2/3 Hydrogen and 1/3 Oxygen, which contains a small percentage of $\mathrm{H}$ and $\mathrm{O}$ magnecular clusters. Under these conditions, Santilli suggested in Ref. [5] the name of HHO (although a similar gas produced via a different electrolyzer is known as Brown's gas). 

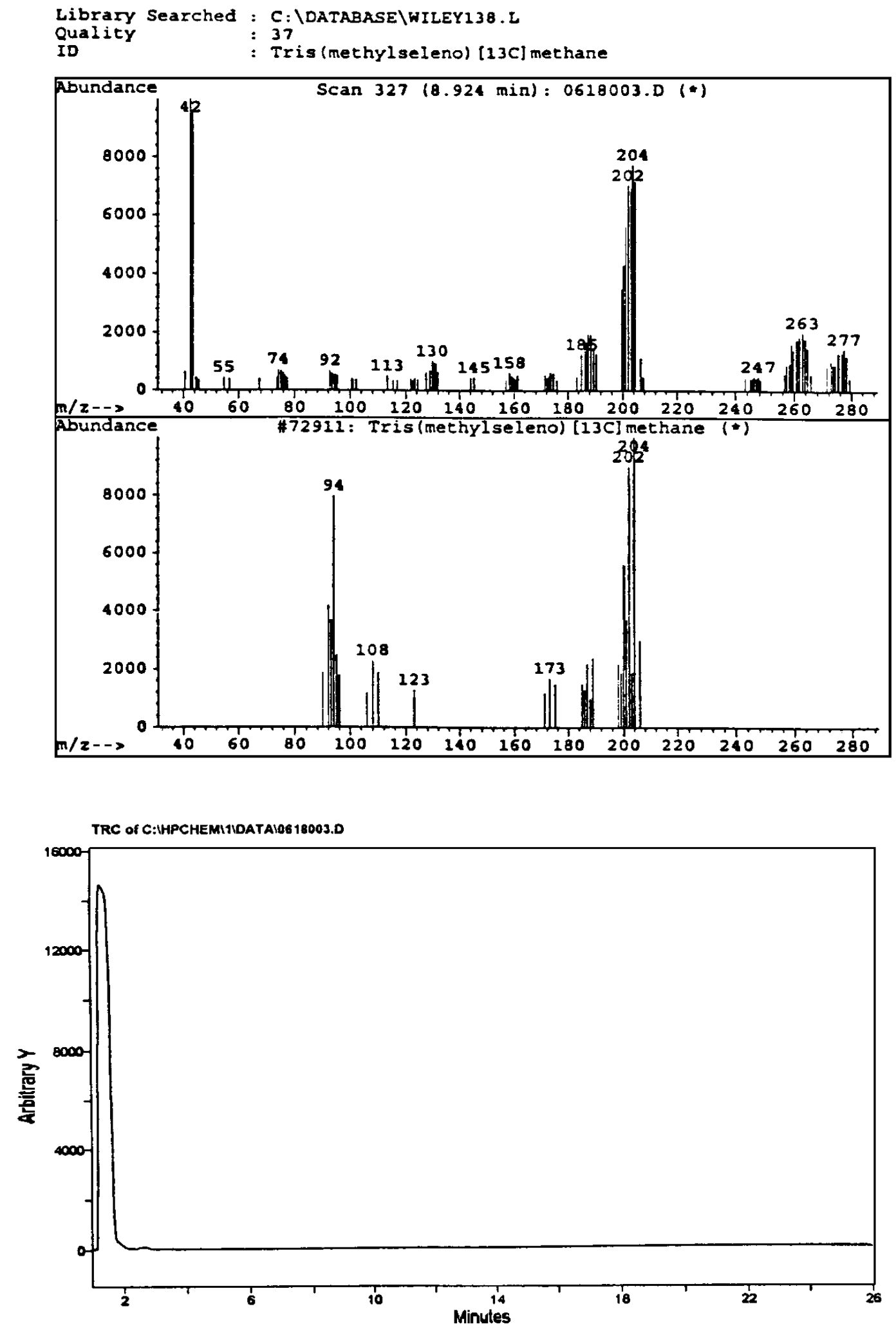

Fig. (4). A representation (top view) from memoir [1] of the first experimental evidence at NTS laboratories that the peaks of (Fig. 3) are "unknown" because of a lack of identification by the computer among 500, 000 known molecules. The same situation occurred for all remaining peaks of (Fig. 3). A reproduction (bottom view) from memoir [1] on the scan of (Fig. 3) obtained via the IRD component of the GCMS/IRD providing the first experimental evidence on the lack of IR signature of the GC-MS peaks of (Fig. 3). In fact, the above IRD scan establishes the existence of large clusters that have no IR signature at all, thus dismissing valence bonds since they would require perfectly spherical clusters that are essentially impossible at large a.m.u. The only identified IR signature is that for the conventional molecule CO2, but the latter refers to the constituents of the peaks of (Fig. 3), because it is not identified as an isolated detectable species. 

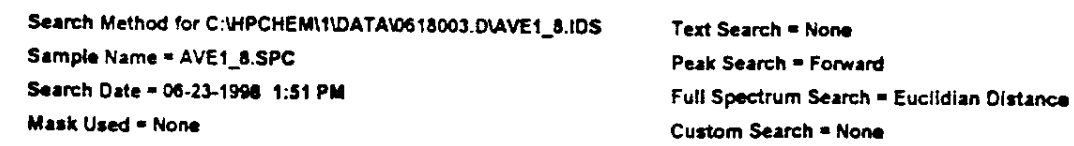

Full Spectrum Search -

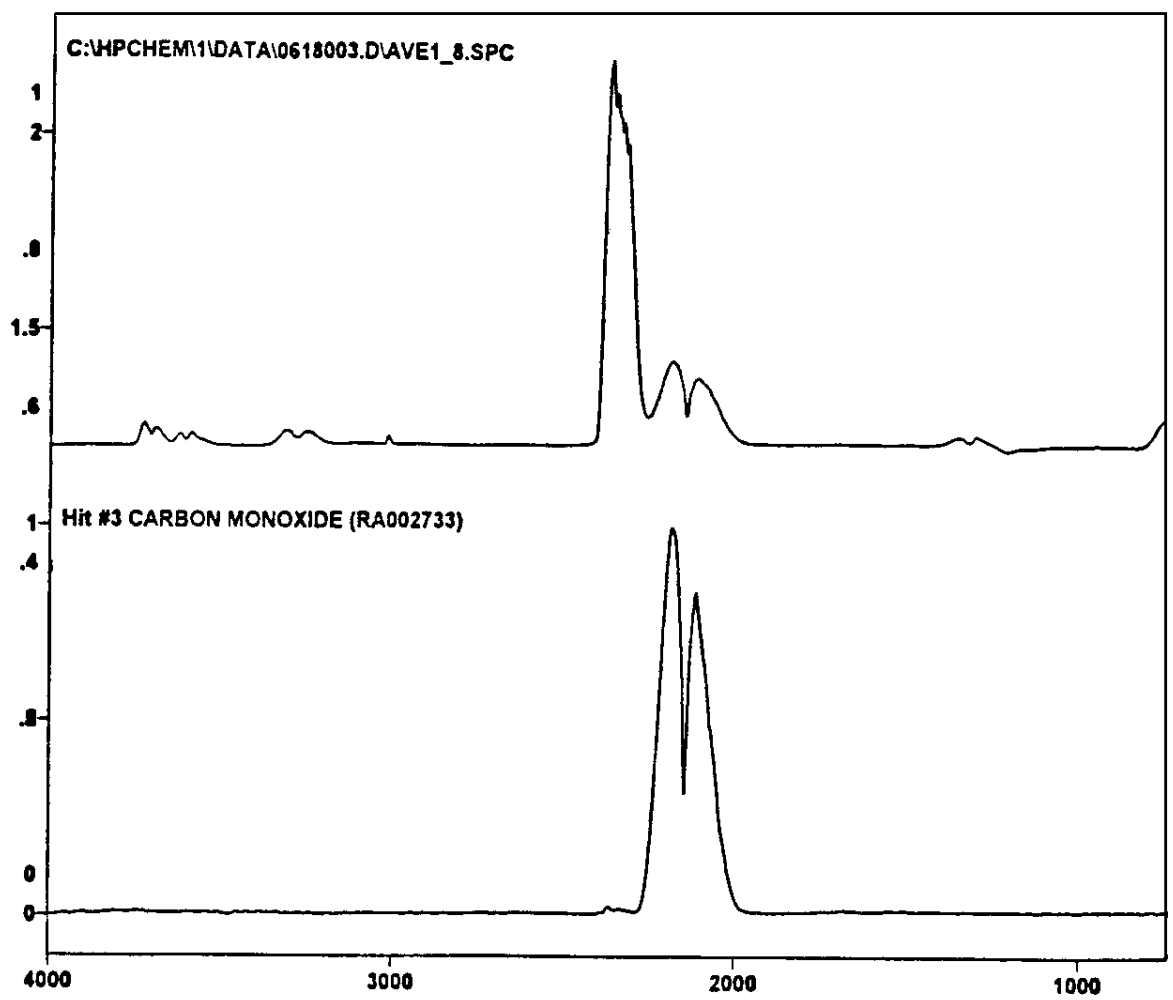

Fig. (5). Reproduction from memoir [1] of the first experimental evidence on the mutation of the IR signature of molecules under a strong magnetic field, here referring to the $\mathrm{CO} 2$ (top) compared to the result of the computer search (bottom). Note that there is the appearance of two anomalous IR signature suggesting new internal bonds, and that the computer erroneously interpreted the molecule as being $\mathrm{CO}$ rather than $\mathrm{CO} 2$.

M. O. Cloonan presented in Refs. [7-9] of 2006 to 2009 applications of Santillimagnecules and its underlying hadronic chemistry to particular forms of pericyclic reactions and related new structures, thus illustrating the expected capability of the new species of Santilli mag- necules to produce new chemical substances (i.e., chemical substances not entirely based on valence bonds).

In monograph [10] of 2008, R. M. Santilli presented an update on the first industrial application of the new species of magnecules via the combustible gas MagneGas indicated earlier, as well as an update of the additional industrial applications for $\mathrm{MH}, \mathrm{MO}$, and $\mathrm{HHO}$.

In Ref. [11] of 2011, R. M. Santilli presented the industrial application for which he developed the new species of magnecules, namely, their use as a necessary premise for the achievement of nuclear fusions without harmful radiation. Santilli's main argument is that preceding attempts at achieving systematic nuclear fusions were flawed by an inability to systematically control the exposure of nuclei out of their electron clouds, the systematic and controlled proper alignment of nuclear spins and other insufficiencies. Inspection of the very conception of a magnecules as in (Fig. 1) illustrates the resolution of these problematic aspects in a systematic, repeatable and controlled way.

The nuclear physicists R. Brenna, T. Kuliczkowski, and L.Ying presented in Ref. [12] of 2011 an independent verification on the existence of Santilli's new fusions without harmful radiation, and confirmed in particular via various chemical analyses the necessary use of magnecular structures for their achievement.

In Ref. [13] also of 2011 Santilli presented additional cases of nuclear fusion, this time for Silica, based on the use of a magnecular bond of the type of (Fig. 1) between Carbon and Oxygen, plus a "trigger," namely, a mechanism pushing nuclei at $1 \mathrm{fm}$ mutual distance at which the strongly attractive nuclear forces are activated and fusion becomes inevitable.

To complete the references on Santillimagnecules, we should additionally quote the second edition of monograph [14] where Santilli presents the application to biology of the new mathematics of increasing complexities known under the names of iso-, geno- and hyper- mathematics. In this monograph, Santilli also shows that most biological struc- 
tures, particularly complex structures such as the DNA, cannot have a molecular structure due to an insufficient number of valence electrons to bond together an extremely large number of atoms. Santilli has introduced the notions of IsoMagneCules, GenoMagneCules and Hyper- MagneCules (also presented in Ref. [4]) to initiate deeper studies on the extreme complexity of biological systems.

In the recently published paper [15], the authors present experimental confirmations of Santilli's new chemical species of MagneHydrogen (MH) and MagneOxygen (MO) originally presented in Ref. [5], which constitute some of the best evidence on the existence of mag- necules, and confirm the anomalous values of their specific weight, energy content and other features.

The work herein presented has been reviewed in Refs. $[16,17]$, while a general review of the mathematical, physical, and chemical aspects underlying the new species of magnecules has been provided by I. gandzha and J. V. Kadeisvili in monograph [18]. This concludes the identification of all published references on Santillimagnecules to to the authors best knowledge at the time of writing this paper (June 2013).

Additional, yet unpublished contributions we believe deserve a mention are: $1>$ Santilli's three independent experimental detections of $\mathrm{H} 3 \mathrm{O}, \mathrm{COH}, \mathrm{CO} 2 \mathrm{H}$ and other anomalous species solely possible under Santillimagnecular bond [19]; 2> The new class of fuels consisting of a magnecular bond of Hydrogen and conventional fossil fuels (such as gasoline, diesel and coal), known as Santilli'sHy-Fuels, with consequential lack of hydrocarbons, $\mathrm{CO}$ and othercontaminants in the exhaust due to their combustion by magnecular Hydrogen [20]; and 3> Santilli's proposal to verify or dismiss experimentally (rather than theoretically) the hypothesis that the Avogadro number increases with the increase of the temperature for gases with magnecular structure [21].

This paper is specifically devoted to outline additional experimental confirmations of the new species of Santillimagnecules as presented in the original memoir [1] of 1998. In the hope of stimulating a collegial participation in this new field of chemistry, the authors will make available to qualified chemists at no cost samples of gases with magnecular structure under the availability of gas chromatographic equipment suitable for the needed measurements.

\section{EXPERIMENTAL CONFIRMATIONS OF SANTIL- LIMAGNECULES.}

The authors have no words to emphasize the difficulties in detecting the new species of Santillimagnecules because, as indicated in the preceding section, all available gas chromatographic equipment has been conceived, developed and tested for the detection of clusters of atoms under the conventional valence bond that, being notoriously strong, allows for strong detection means (such as ionization, thermal conductivity. etc.) without destroying the species to be detected.

By contrast, magnecular bonds are much weaker than valence bonds by conception and industrial realization, so as to allow full combustion $[4,10]$. Therefore, detection equipment and procedures that are unquestionably valid for molecules, may in reality destroy the very magnecular species to be detected, unless appraised with care, caution and objectivity.

One of the difficulties experienced by Santilli in the decade long failed attempts at reproducing the measurements presented in memoir [1] is the existence of over 750, 000 conventional molecules that have been identified so far. Whenever a chemical species is identified in a GC or a GCMS, it is rather natural for experienced analysts to assume that it merely consists of either a known conventional molecule, or a molecule yet to be identified, thus denying the possibility for chemical novelty. Consequently, following memoir [1], a decade of testing gases with magnecular structure at various analytic laboratories around the word turned out to be scientifically sterile.

Another difficulty encountered by Santilli has been caused by the understandable tendency of senior analysts to assume that any chemical anomaly (that is, novelty over established 20th century knowledge) is due to a malfunction of the instrument, in which case all efforts are generally made to modify the operation of the instrument (by increasing the column temperature, decreasing the elusion time, etc.) until conventional results are obtained without any chemical novelty.

Further difficulties are encountered by Santilli are created by the fact that the chemical novelties of magnecular species are generally dismissed a priori by analysts, thus preventing their serious experimental confirmation or dismissal. As an example, magnecular gases have an anomalous adhesion to all substances, including paramagnetic ones (because magnetization by induction occurs at the atomic, rather than molecular level). Following a test with a GC-MS and a conventional flushing of the instrument, the background retains most of the peaks detected during the normal test $[1,10]$.

Analysts generally consider this occurrence a malfunction of the instrument, and often send it to the manufacturer for service, rather than admitting an essentially new chemical occurrence deserving inspection. In reality, the conventional background is readily recovered by flushing the instrument with a hot inert gas (such as Nitrogen at $400^{\circ} \mathrm{C}$ ), by confirming in this way the sensitivity of magnecular species to high temperatures, as expected for all magnetic polarizations.

Unsurmontable difficulties were additionally experienced by Santilli in detecting mag- necules with the recent generation of gas chromatographic equipment using capillary feeding lines, because they rapidly clog up following the injection of a magnecular gas due to its anomalous adhesion, thus providing the analyst the mere illusion of analyzing the gas, while in reality the gas to be tested did not reach the column in the necessary volume.

In one case, Magnegas Corporation had to hire attorneys to have a major U. S. analytic laboratory equipped with the most advanced GC-MS to dismiss its signed report on the commercially produced and sold MagneGas ${ }^{\mathrm{TM}}$ fuel (see www.magnegas.com for details). In essence, the analyst calibrated the GC-MS with air, flushed the instrument via established procedures, injected MagneGas in the capillary feeding line, conducted a variety of measurements, and released a written and signed report according to which MagneGas was contaminated at least $30 \%$ air in volume (since 
the scans showed the signatures for Nitrogen and Oxygen in the indicated volume and correct proportions).

Magnegas Corporation is a publicly traded company at NASDAQ (with symbol MNGA) and under SEC scrutiny sells MagneGas as a fuel. Therefore, its contamination of the fuel with $30 \%$ air would render it explosive, thus in violations of civil and criminal laws. These implications mandated the hiring of attorneys for the dismissal of the signed report, which dismissal was indeed achieved via certified analyses of the same gas but tested with an older GC-MS having a macroscopic feeding line. This episode confirmed that capillary feeding lines are clogged up by gases with magnecular structure, thus prohibiting the entrance in the instrument of the very species to be detected.

When the authors became interested in an independent confirmation or dismissal of the new species of Santillimagnecules, they became aware of Santilli's decade of fruitless attempts at a confirmation of the species with gas chromatographic equipment available at commercial and academic laboratories, and conducted a study for the identification of the equipment and its use for the detection of magnecules. The authors conclusion is that the best way currently possible to detect magnecular species is via a GC-MS/IRD as originally used by Santilli in 1998 [1].

We are here referring to the principle of jointly testing the same gas with two different equipment, the GC-MS for the identification of the clusters composing the species, and the IRD for the verification as to whether the bond responsible for the clusters is of valence type or not. In particular, a molecular interpretation should be accepted not only when the clusters at the GC-MS are identified by the computer as being known molecules, but also when their known IR signature is confirmed in the IRD "at the a.m.u. value of the cluster," and not at smaller a.m.u. values, since the latter refer to the conventional "constituent" of the cluster. On the contrary, when clusters admit no IR signature at their a.m.u. value, then said clusters cannot possibly be formed by a valence bond since that would require a perfect spherical shape at large a.m.u. values which is known to be a physical and chemical impossibility.

Due to the protracted difficulties experienced by Santilli with contemporary equipment, the authors secured exactly the same instrument used by Santilli in 1998 [1], namely, a GC-MS/IRD consisting of a HP GC model 5890, a HP MS model 5972, and a HP IRD model 5965 equipped with a HP Ultra 2 column $25 \mathrm{~m}$ long, $0.32 \mathrm{~mm}$ ID, and film thickness of $0.52 \mathrm{um}$, with temperatures starting at $100 \mathrm{C}$ for $4 \mathrm{~min}$, then raising to $2500 \mathrm{C}$ at $10^{\circ} \mathrm{C} / \mathrm{min}$.

The production and service of the above identified GCMS/IRD was discontinued one decade ago. Therefore, the desired instrument had to be restored from an old one. Such a restoration was commissioned by Spectral Scientific Incorporation (SSI) 38 McPherson Street, Markham, Ontario, Canada. The authors exercised particular supervision during this restoration to prevent the expected tendency to "upgrade" the instrument, which upgrade would have likely prohibited the desired measurements as well as the appearance of this paper. Following about two years of laboratory work, the fully restored and operational GC-MS/IRD was delivered by SSI in early 2012, and tests initiated immediately thereafter.

The following additional instrument was needed to achieve a comprehensive verification or dismissal of Santilli's measurements from 1998 [1]. In essence, the chromatographic equipment that had systematically dismissed the existence of the new species of magnecules is the GC-TCD. However, contemporary GC-TCD is definitely not recommendable for serious analyses of magnecular gases due to the use of capillary feeding lines and undesired detection procedures.

Consequently, the authors selected an old GC-TCD comprising HP model 5890-2T and a HP GC model 5890 Series II equipped with two columns, one being a packed- column $80 / 100$ mesh, and the other being a molecular-Sieve 5A Column. The authors then requested its restoration to Global Medical Instruments, Inc. 6511 Bunker Lake Blvd, Ramsey. MN., U.S.A. Again, special attention had to be devoted to prevent damaging "upgrades" during the restoration. Following about one year of laboratory work, the desired instrument was delivered also in full operational conditions in early 2012, and tests were initiated immediately thereafter.

Amongst a large number of additional chemical analyses of magnecular gases accumulated by Santilli in a decade of studies, we have selected a representative scan obtained by Oneida Research Services, 8282 Halsey Rd, Whitesboro, NY, via IVA 110 s equipped with a vacuum chamber (with an air-cooled turbomolecular pump), sample inlet with temperature control system and monitor, high performance quadrupole mass spectrometer system, interchangeable electro-pneumatic and manual sample piercing system, electropneumatic vacuum isolation valves, inlet pressure monitor for pump down, automatic calibration port, computercontrolled sampling valve and valve switching panel (VSP).

The used IVA 110 s has a system sensitivity better than 100 ppmv for moisture and better than $10 \mathrm{ppmv}$ for other gases and a system accuracy of $\pm 5 \%$ at 5000 ppmv. Also, the IVA 110s utilizes a NIST (National Institute of Standards and Technology) Mass Spec Database of more than 250,000 spectra with IVACS interface for use in identification of unknowns.

Following initial doubts due to their novelties under consideration, our analyses confirm all of Santilli's claims presenetd in memoir [1] for the case of gases with magnecular structure without exceptions known to the authors. Out of a large variety of tests with different magnecular gases, different equipment and different procedures, our main results are presented in the representative scans of in (Figs. 6 to 19), and can be summarized as follows:

1. Characterization of magnecules by weakly bonded individual atoms, dimers, and conventional molecules;

2. Stability of magnecules at ambient temperature;

3. Progressive reduction of magnecules with the increase of the temperature;

4. Termination of magnecules at a suitable Curie temperature;

5. Detection of magnecular clusters under a suitably selected and operated GC-MS; 

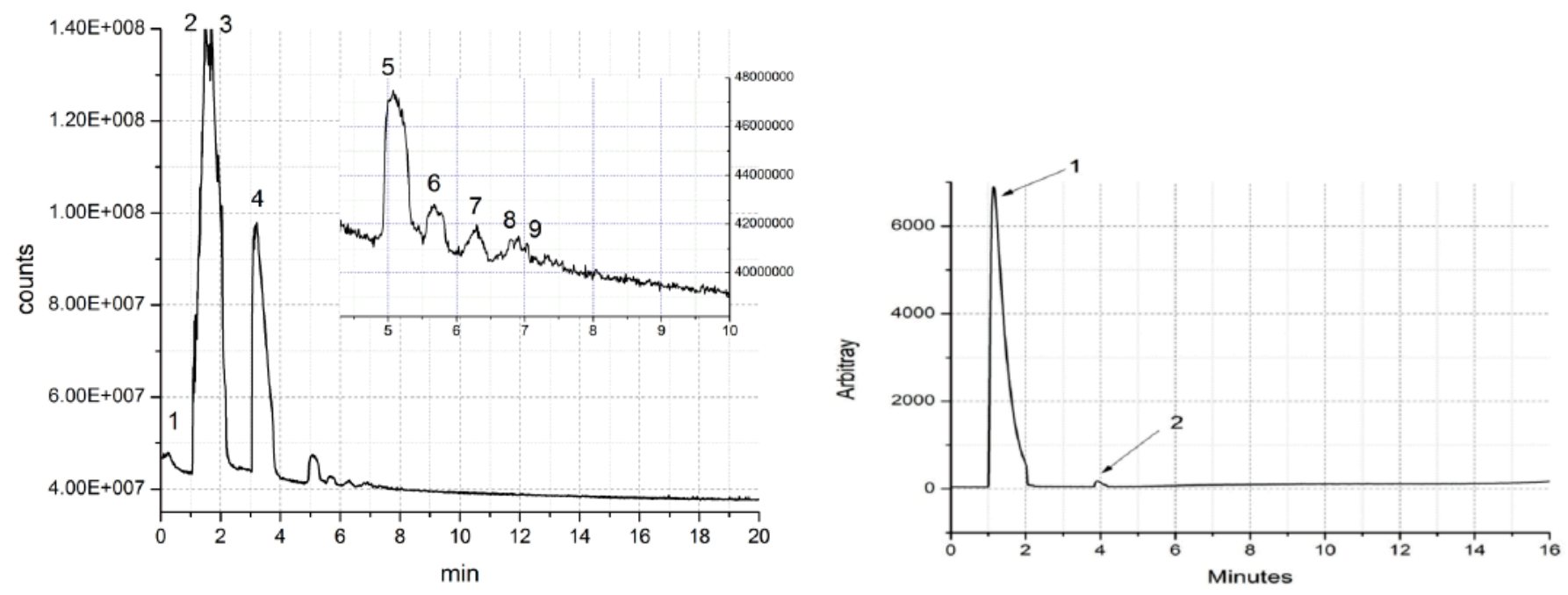

Fig. (6). Representative scans achieved by the authors on November 8, 2012, via the GC- MS/IRD described in the test. The top view presents a GC-MS scan from 1 a.m.u. to 500 a.m.u. with the column operated at $100 \mathrm{C}$ and the use of 22 minutes elusion time. The tests were conducted on filtered and compressed MagneGas synthesized from ethylene glycol. In the bottom view, we present the IRD of the same gas and the same injection which provides experimental confirmation for the presence in MagneGas of clusters without an IR signature, thus confirming the main claim of memoir [1], namely, the existence in nature of bonds other than those based on valence. Note that the MagneGas used for the scans here is different that used for the tests of Memoir [1], as confirmed by the fact that the peaks of (Fig. 4) are different than those of this figure.
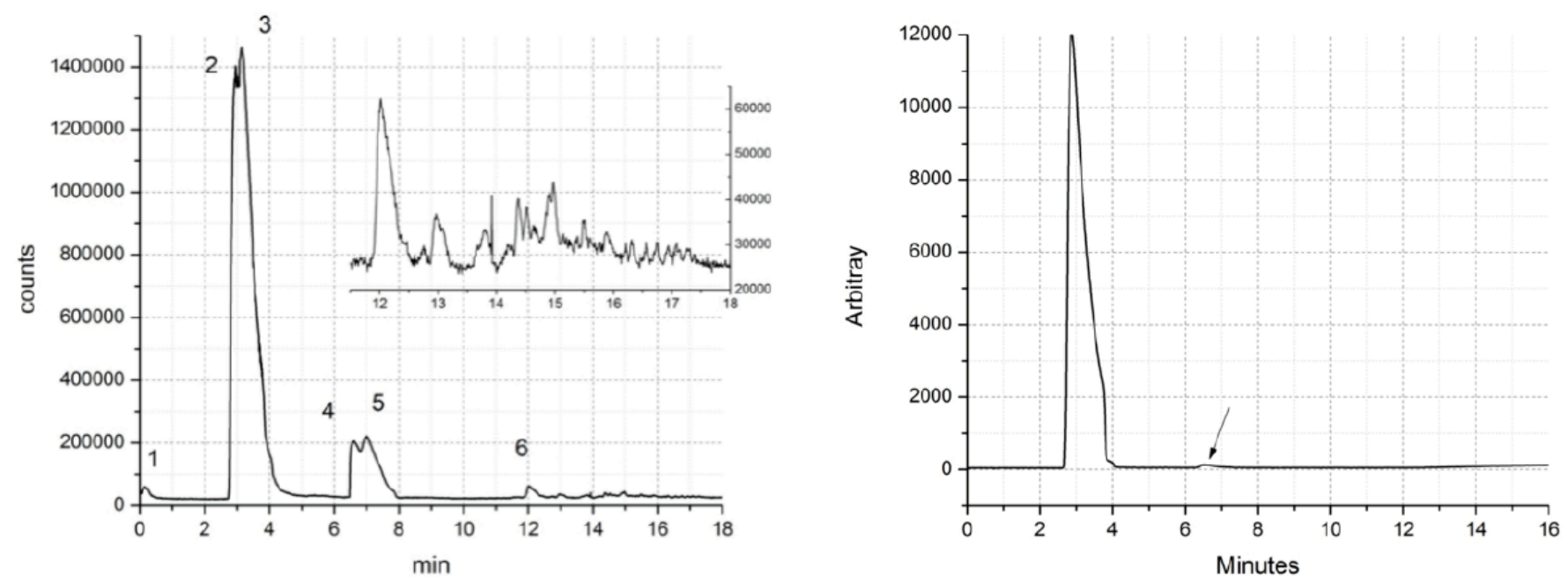

Fig. (7). Scans obtained via the same GC-MS/IRD, the same 22 minutes elusion time, and the same magnecular gas as those for (Fig. 6), but with the column operated at $400 \mathrm{C}$. In the top view, we present a GC-MS scan showing a decrease of the magnecular species of (Fig. 6) due to the increased temperature of the column, thus confirming the dependence of the detection of magnecules from the column temperature first identified by Santilli [1]. In the lower view, we present an IRD scan of t1h2e same setting as in the top view confirming the absence of IR signature by magnecular clusters also identified in Ref. [1]. Additional comments are presented in the text.

6. Transparency of magnecules to infrared detectors for the a.m.u. of the clusters (and not at a smaller a.m.u. characterizing constituents);

7. Dependence of detected magnecules from the elusion time;

8. Dependence of magnecular species from filtration and compression;

9. Anomalous adhesion of magnecular gases to disparate materials;

10. Anomalous mutation of magneculer clusters in time and under different detection pro- cedurtes or equipment;
11. Anomalous accretion of magnecular clusters by individual atoms; and other features.

A few comments are in order.

Property 1 [1] is confirmed by our various measurements as follows. The weak character of magnecular bonds is defined with respect to the strength of valence bonds in molecular gases. Said weak character is then confirmed by the lack of hydrocarbons, $\mathrm{CO}$ and pollutants in the exhaust of Santillimagnegases compared to the pollutants existing in the exhaust of molecular fuels, such as gasoline. (Figs. 12 to 17) establish that magnecules contain conventional dimers and molecules under the indicated weak magnecular bond. The 

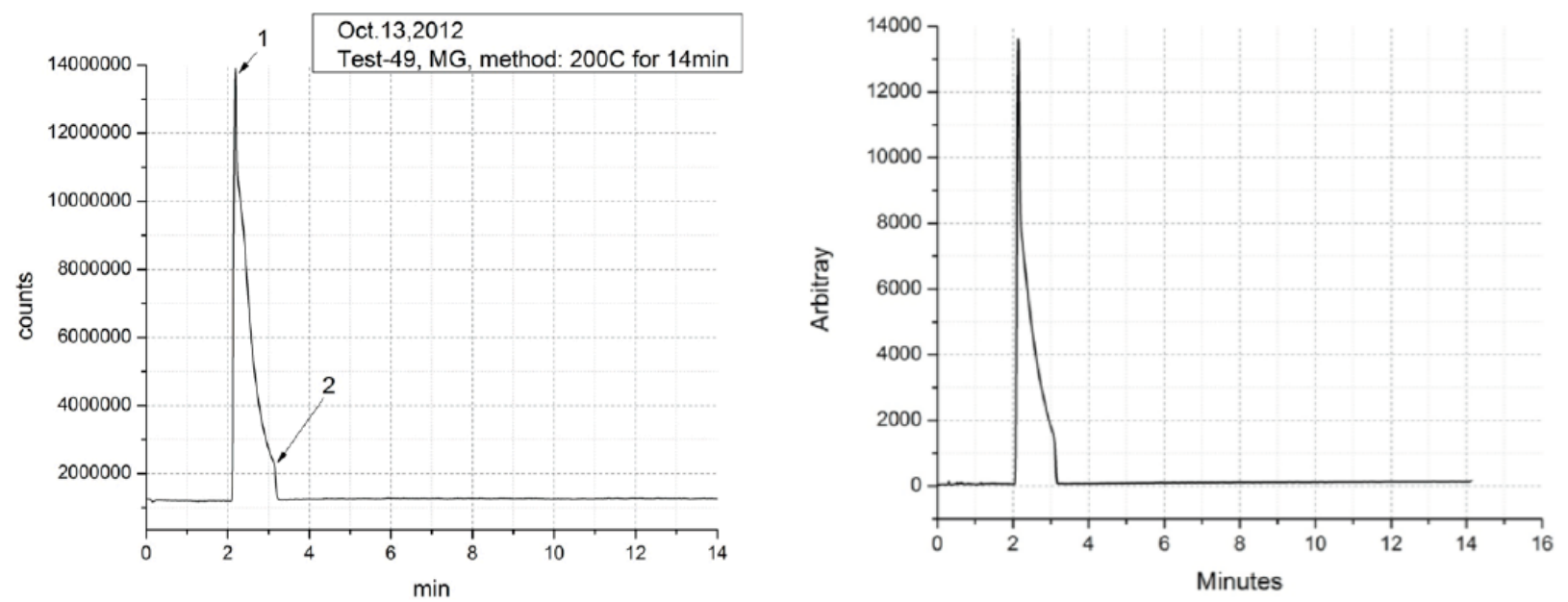

Fig. (8). Scans obtained via the same GC-MS/IRD, the same 22 minutes elusion time, and the same magnecular gas as those for (Fig. 6), but with the column operated at $200^{\circ} \mathrm{C}$. The top view presents a GC-MS scan showing the disappearance of magnecules, thus confirming Santilli's claim [1] that magnecular clusters admit a temperature (the Curie temperature) at which magnetic bonds disappear. The lower view presents the corresponding IRD scan confirming the absence of IR signatures for magnecular species [1].
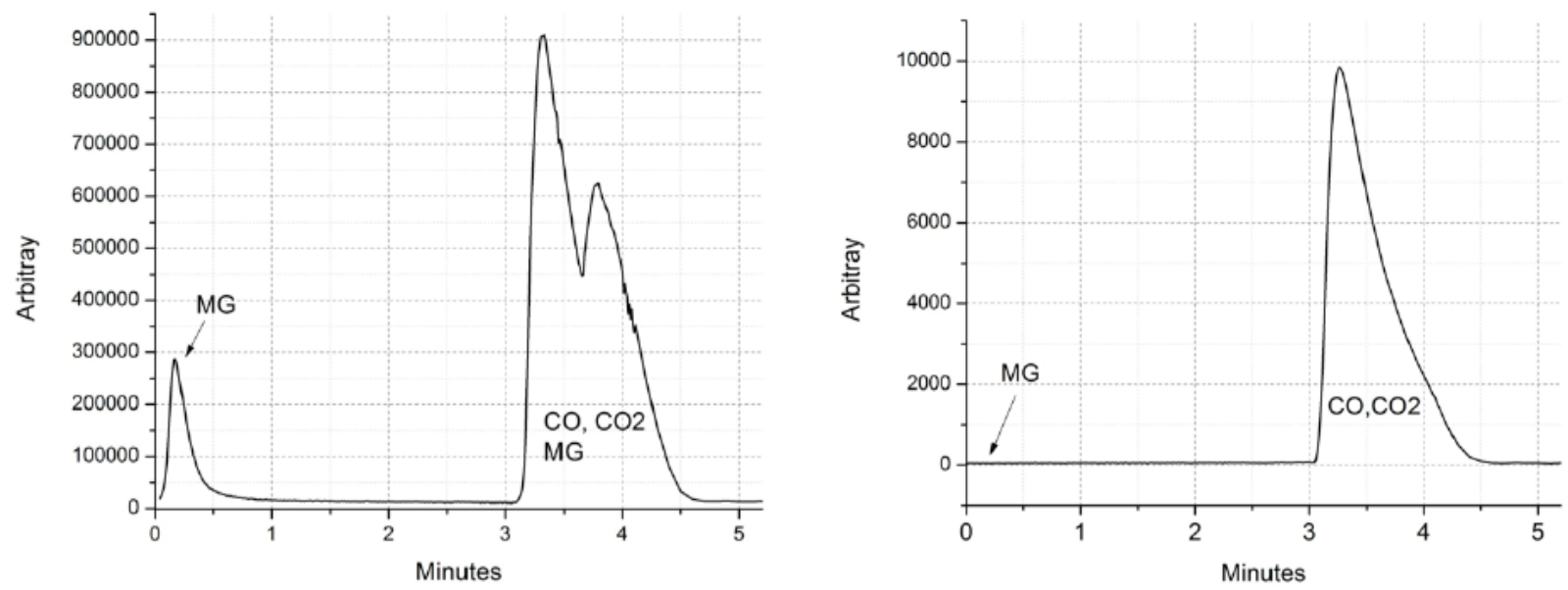

Fig. (9). Scans obtained via the same GC-MS/IRD, and the same magnecular gas as those for (Fig. 6), but with the elusion time reduced from 22 to 5 minutes and the column temperature increased from $10^{\circ} \mathrm{C}$ to $55^{\circ} \mathrm{C}$. The top view confirms Santilli's mutation of magnecular clusters [1], namely, their clear change with the change of procedures in the same instrument. The bottom view presents an IRD scan that also shows deviations from the corresponding scan of (Fig. 6), again in agreement with Ref.[15].
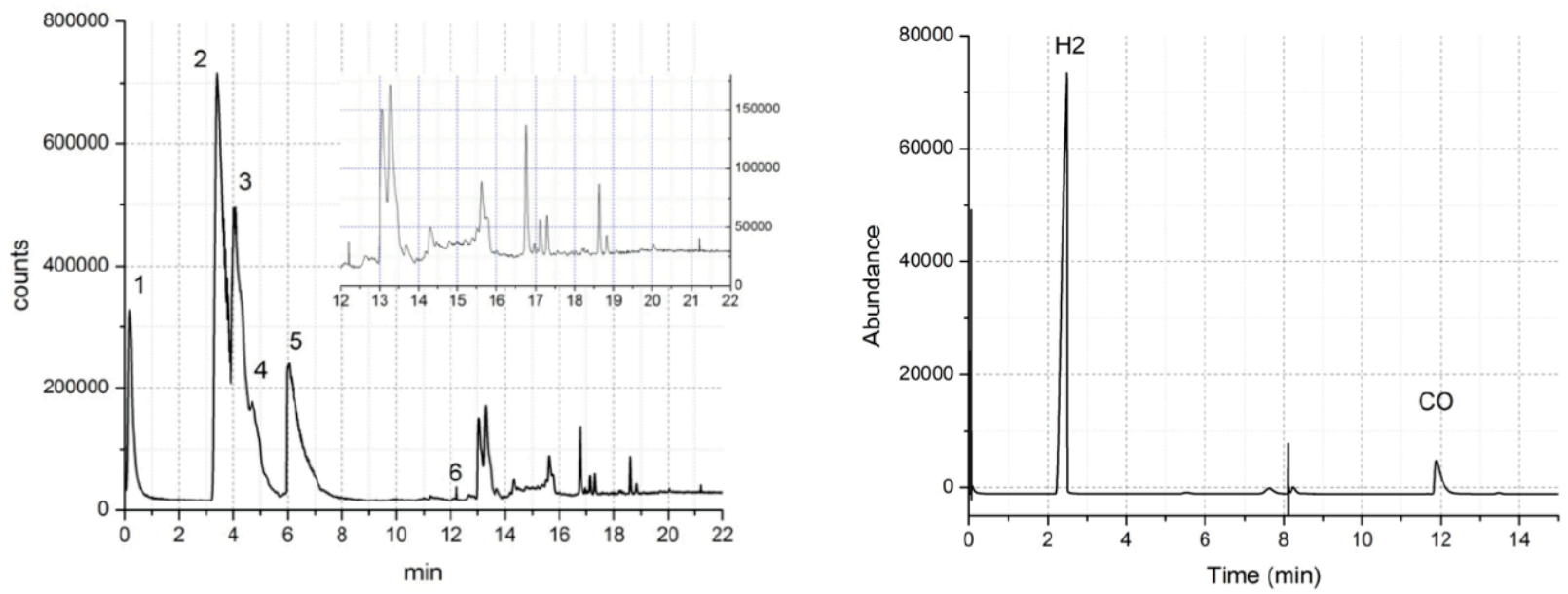

Fig. (10). In the top view, we show a GC-MS scan at $10^{\circ} \mathrm{C}$ of MagneGas synthesized from ethylene glycol collected prior to filtration and compression. The scan illustrates the loss of magnecular species during filtration and compression, due to the appearance in the above scan of anomalous clusters that are absent in the scans of (Figs. 6-9). The bottom view shows an IRD scan confirming the preceding onces. 


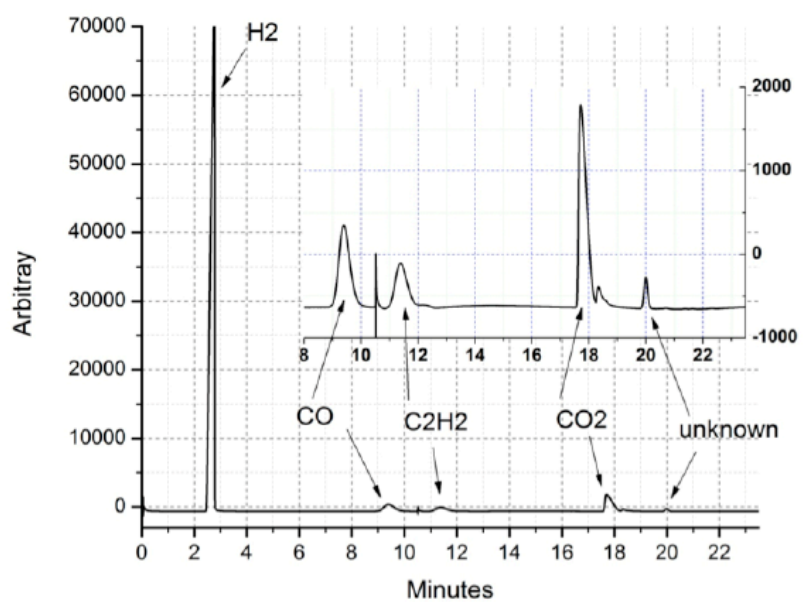

Fig. (11). Scan of MagneGas of (Fig. 6) with the GC-TCD identified in the text and operated from $400 \mathrm{C}$ to $2000 \mathrm{C}$ showing the conventional molecular constituents of MagneGas without any appreciable magnecules. This confirms Santilli's claim [1] that GC-TCD operated at temperatures normal for molecular species destroys, rather than detects, magnecular species.

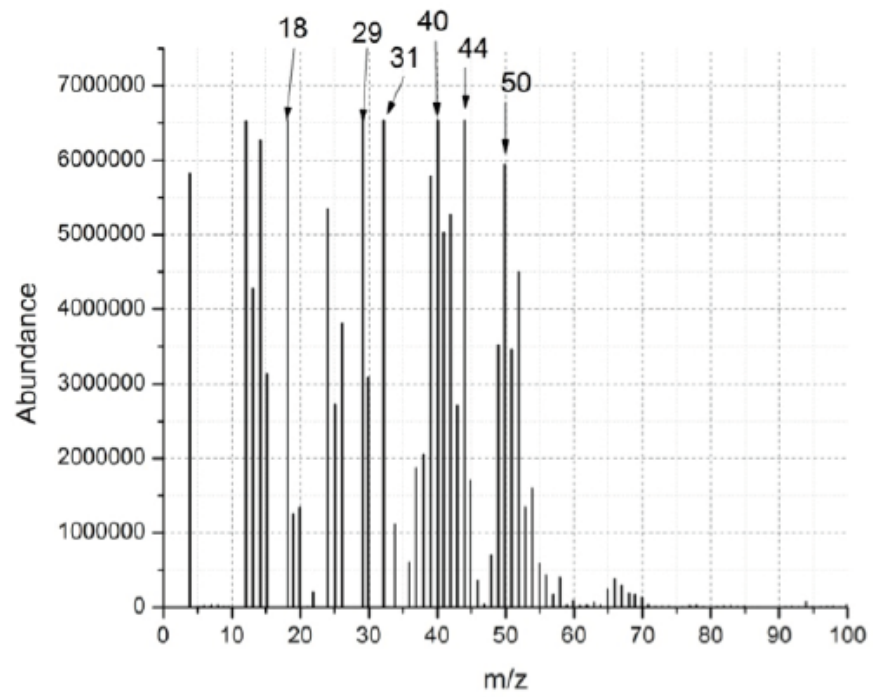

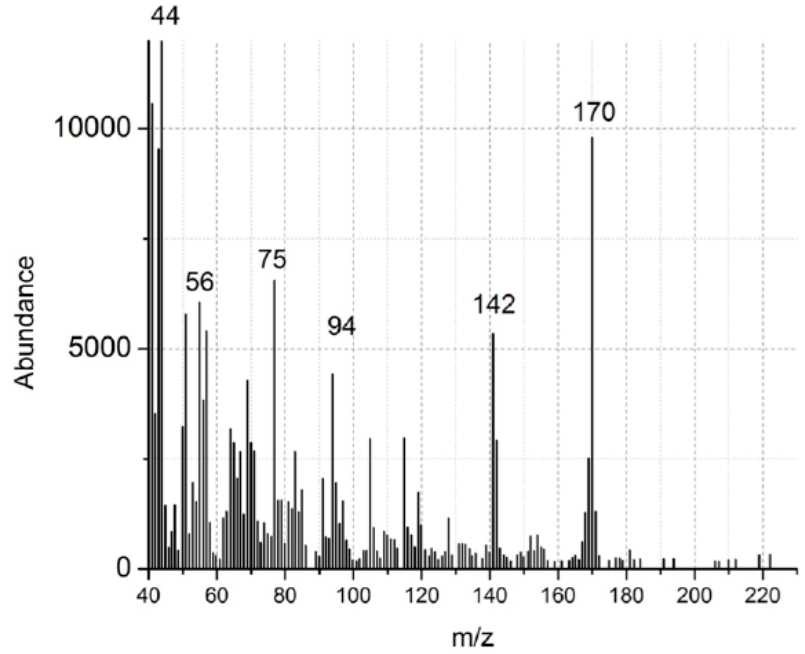

Fig. (12). MS scan of peak 1 of the GC-MS view of (Fig. 6) showing the presence of conventional molecules as constituents of the peak, plus new magnecular species, all bonded together in peak 1 of (Fig.6).

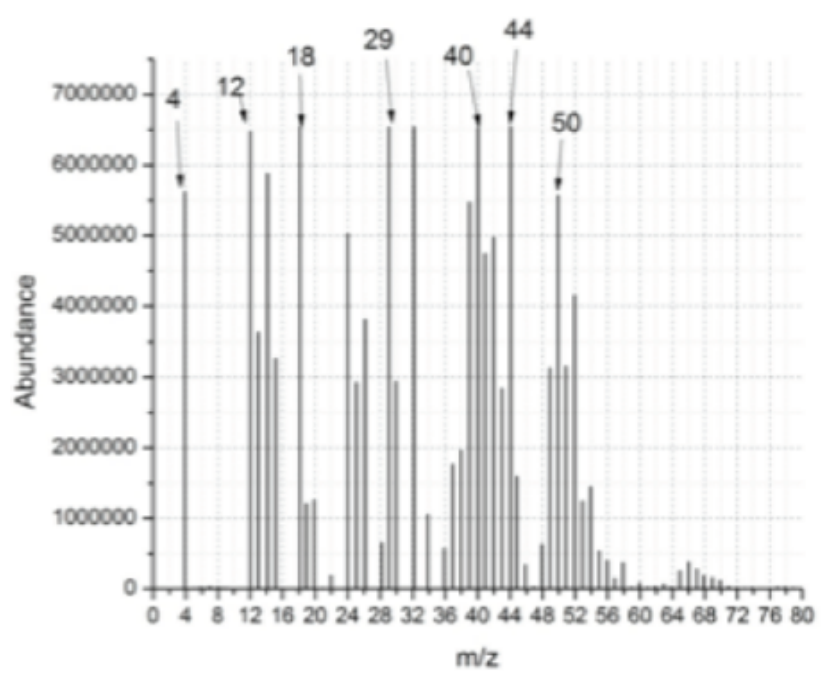

Fig. (13). MS scan of peak 2 (top) and 3 (bottom) of the GC-MS view of (Fig. 6) confirming the mixed nature of Charlemagne clusters.
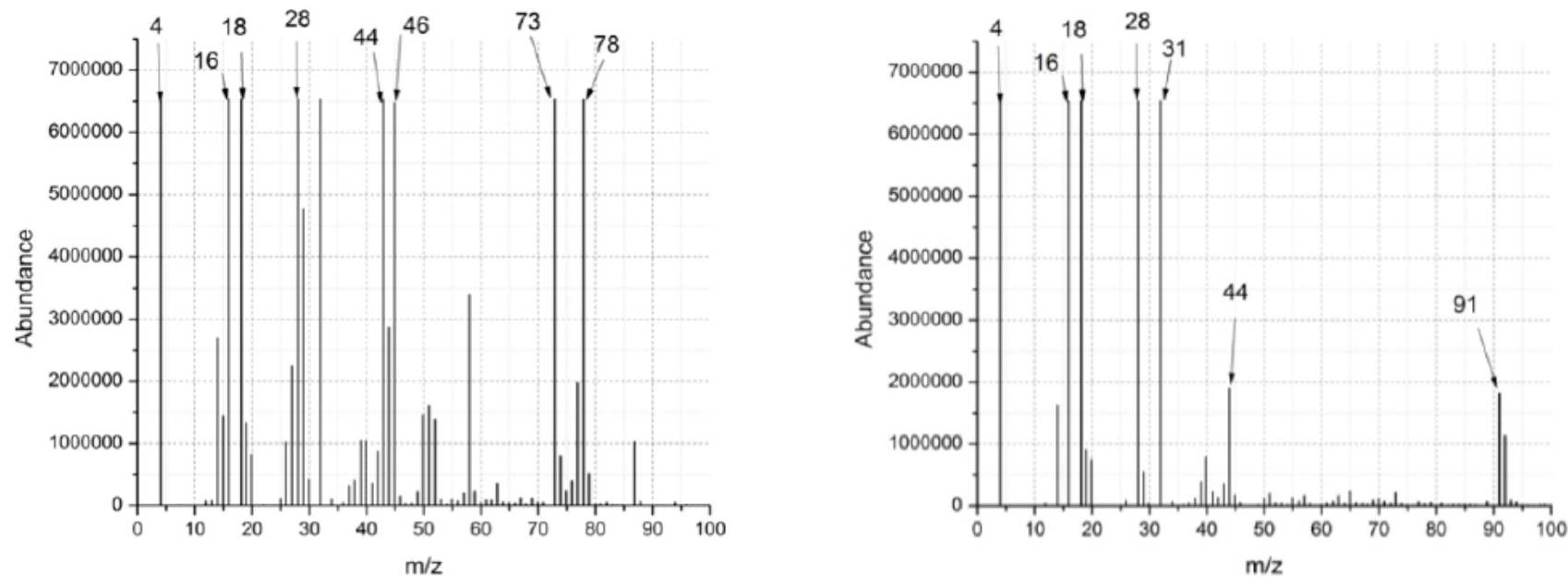

Fig. (14). MS scans of peaks 4 and 5 of the GC-MS view of (Fig. 6). 

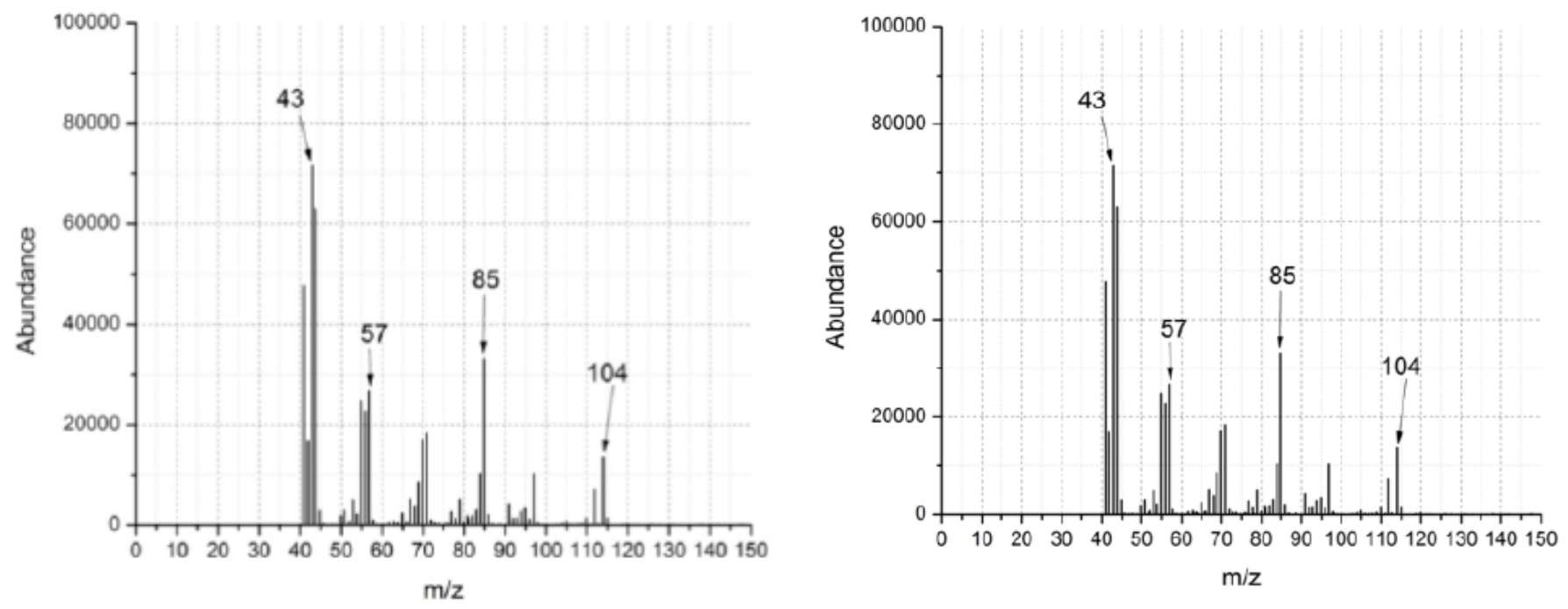

Fig. (15). MSscans of peaks 6 and 7 of the GC-MS view of (Fig. 6).
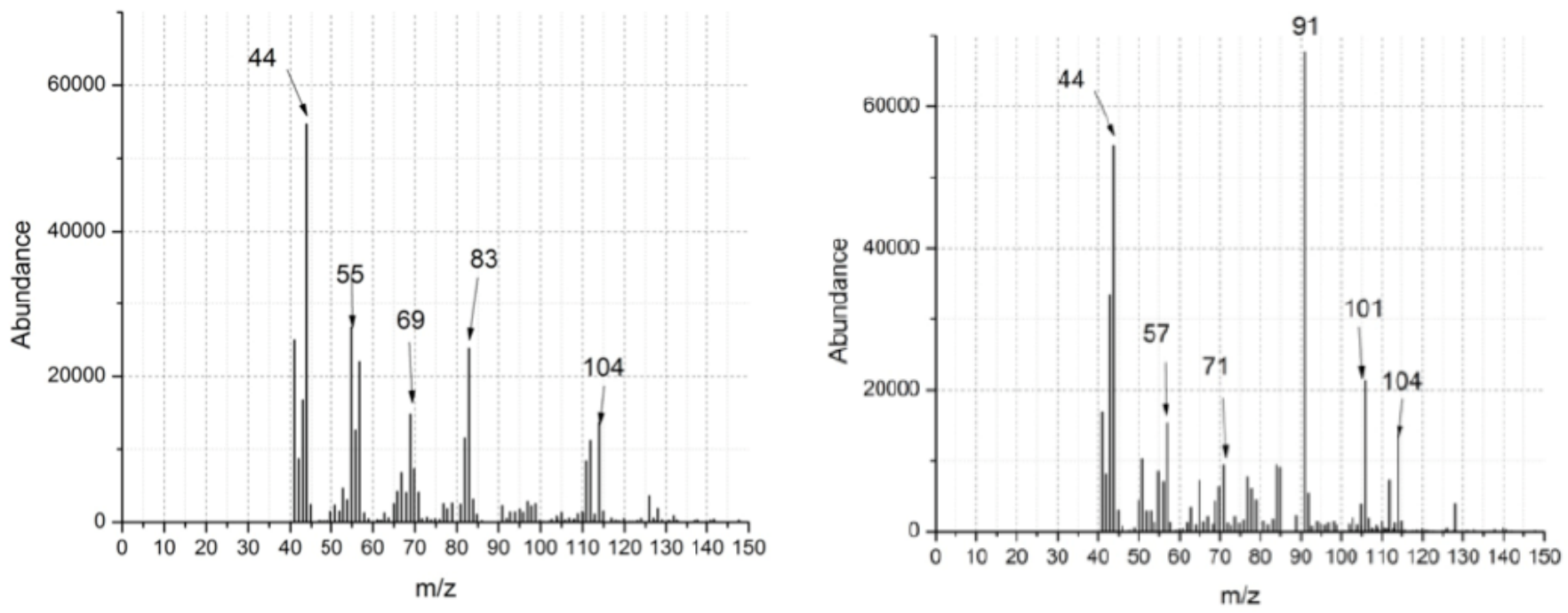

Fig. (16). MSscans of peak 8 and 9 of the GC-MS view of (Fig. 6).

presence in magnecules of individual atoms under weak magnecular bond is established for the case of Hydrogen by the scans of (Fig. 18) showing Santilli's accretion of magnecules by 1 a.m.u., with similar accretion for other atoms.

Property 2 [1] is established by data of the U. S. publicly trade company Magnegas Corporation with website www.magnegas.com (founded and directed by Santilli until recently), which company has been selling magnegas for various fuel uses in pressure bottle up to 5,000 psi that have been kept at time in storage at ambient temperature for years without any change of pressure, combustion and exhaust.

Property 3 [1] is illustrated by the comparison of (Figs. 6 and 7) because the decrease of magnecules with the increase of the temperature is detectable with the sole increase of the column temperature from $10 \mathrm{o} \mathrm{C}$ to $40 \mathrm{o} \mathrm{C}$. This is another confirmation of the weak character of magnecular bonds according to Santilli's original conception [1] because necessary to achieve full combustion. In essence, magnecular clusters routinely break down and then recombine due to thermal collisions (a feature absent for molecular gases due to the

strength of their valence bond). Then, their recombination evidently decreases with the increase of the temperature, that is, with the increase of the collisions.

Property 4 [1] is established in various ways, such as via the measurements provided by Magnegas Corporation according to which there is no trace of magnecules whatsoever in the exhaust of magnecular fuels. Therefore, as also predicted in memoir [1], the combustion temperature of magnecular fuels is their Curie temperature.

Property 5 [1] is confirmed by the numerous scans reported in this paper which have been obtained via a suitable GC-MS operated under special requirements, such as at the lowest possible column temperature, the longest possible elusion time, cryogenic cooling of the feeding lines and other requirements identified by Santilli $[1,4]$. A basic aspect is that the first step in the experimental detection of magnecules requires the study of clusters detected by the GC-MS "at their a.m.u. value," and not the usual study of their reduction to conventional constituents. Consider, for instance, peak 1 of (Fig. 6). Conventional analyses reduce that peak to 
its constituents as in (Fig. 12), resulting in no detection of magnecules at all. In reality, clusters of conventional molecules cannot be detected in the GC-MS at a given, generally large and repeatable a.m.u. value unless there is a form of bonding the molecular constituents together. The admission of this chemical reality is the birth of Santilli magnecules [1] because it is evident that conventional valence bonds cannot possibly bond ordinary molecules into clusters detectable in GC-MS thus requiring a non-valence interpretation.

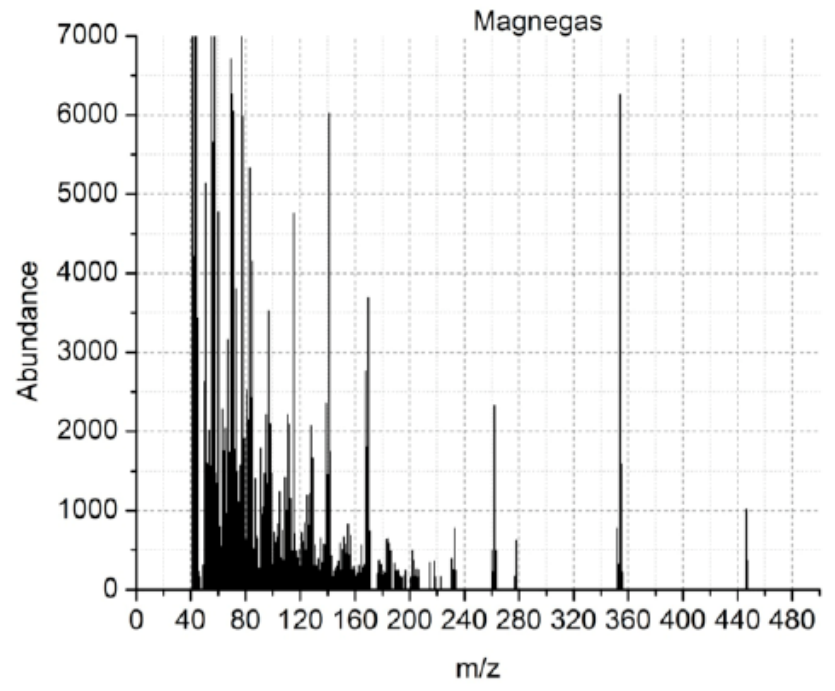

Fig. (17). MS scansofpeakMG of (Fig. 9) confirming the presence of magnecular constituents.

Property 6 [1] is the second step in the experimental detection of magnecules. In fact, the detection of a cluster at a given a.m.u. value in the GC-MS that does not correspond to any known molecule (see, e.g., the top of Fig. 4) is not sufficient scientific evidence to establish that such a cluster is a magnecule, thus requiring at least another analysis. The only second independent analysis available at the time of the discovery of magnecules in 1998 [1] as well as now, is the IRD, provided that it is attached to the GC-MS to avoid the ambiguities indicated earlier. Consequently, a cluster of over 2 a.m.u. in the GC-MS is then confirmed as being a magnecule when said cluster has no IR signature at the a.m.u. value of the cluster and not of its constituents $[1,4]$. To understand the importance of this point we should perhaps quotes Santilli in his lectures at www.world-lecture-series.org: In the absence of two, independent and confirmatory measurements, I consider claims of identification of a given molecule via one single analysis as being the personal opinion of the analyst, and certainly not a final scientific reality. In order to prevent sending innocent people to the electric chair, forensic laboratories are requested by law to use two independent confirmatory analytic measurements for any chemical identification. It is unfortunate that such a sound scientific practice is not generally used in contemporary academic laboratories.

Property 7 [1] is clearly confirmed in the various scans presented in this paper. The property indicates that some of Santillimagnecules can be created by the very instrument used for their detection, according to a mechanism discussed in details in the creation of the new species of SantilliMagne Hydrogen (MH) [15]. To avoid repetitions, we merely men- tion that under a sufficiently long elusion time, magnecular clusters have sufficient time to acquire an axial symmetry, thus align themselves inside the column and then bond to each other under pressure in the confined cylindrical area of the column, thanks also to contributions from internal stray fields in the interior surface of the column.

Property 8 [1] is also confirmed not only by the scans presented in this paper, but also by the industrial data provided by Magnegas Corporation in the website www.magnegas.com. Consider, as an example, the industrial production of Santillis $M H$ now under way, which consists of cluster of Hydrogen molecules and individual Hydrogen atoms under magnecular bonds with structure given by a combination of the following values $\mathrm{MH}=\{2,3,4,5,6$, $7, \ldots$, a.m.u. $\}$. It is evident that Pressure Swing Adsorption and other equipment achieving a filtration all the way to 2a.m.u. would push the filtration to the limit of eliminating altogether the new species of $M H$. Compression may also be an additional reason for the reduction of magnecules, because of the partial catalytic liquefaction of magnecular gases under pressure when exposed to the various metals present in compressors that act as catalysts.

Property 9 [1], called by Santilli anomalous adhesion, was clearly established in the original discovery of 1998, confirmed in the various measurements presented in this paper, and routinely verified in the industrial production and use of magnecular gases. A good illustration is that GC-MS often show serious malfunctions after testing magnecular gases followed by routine flushing with an inert gas. The malfunctions can at times be such that various analysts send out the GC-MS for service. In reality, the GC-MS reacquires full operational capabilities via mere flushing with an inert gas at $400^{\circ} \mathrm{C}$. The most plausible interpretation known to the authors is the original one [1], namely, that magnecules attach themselves via magnetic induction to the internal parts of the detector, and such an anomalous bond is broken at a sufficiently high temperature. An important novelty is that Property 9 occurs irrespective of whether the adhesion occurs to a paramagnetic substance or not, because the magnetic induction occurs at the atomic (rather than molecular) level for which all electron orbitals can be polarized. It is significant to quote Santilli as the leading scientist in magnecules when he states in his works [4]: A good practical way to verify that we are dealing with magnecules is to flush the GC-MS with an inert gas at low temperature. In the event the blank after such a flushing shows clear clusters, then they can only exist due to anomalous adhesion that, in turn, is a clear signature of a magnecular gas.

Property 10 [1], called by Santilli anomalous mutation, can be best illustrated by the fact that the analysis via a GCTCD operated at high temperature eliminates all detectable magnecules. As established in the original proposal [1] and confirmed in this paper, a series of magnecular clusters' may change in time in a GC-MS when there is no variation at all in the instrument and in the detection. The change generally occurs for Santilli accretion, or loss, of one or more atoms. Evidently, this anomalous mutation is a consequence of the other features, such as the continuous breaking down of magnecular clusters and their recombination mentioned earlier. The detection of different magnecules by different gas 
chromatographers is evident and needs no additional comments.

Property 11 [1], called by Santilli anomalous accretion, consists in the capability of magnecular clusters (only) to acquire one or more polarized Hydrogen atoms. This property is established by numerous scans of Refs. [1,4-6] as well as those of (Fig. 18) of this paper, and three independent measurements presented in paper [19] on the detection of $\mathrm{H}_{3} \mathrm{O}, \mathrm{COH}, \mathrm{CO}_{2} \mathrm{H}$ and other anomalous species that are stable at ambient temperature. Property 11 identifies features with important environmental implications when realized via Santilli magnecular fuels. As an illustration, said property allows the industrial development of a new class of clean burning fuels proposed by Santilli under the name of HyFuels [20], which consist of a stable (at ambient temperature) magnecular bond of Hydrogen and fossil fuels, such as gasoline, diesel or coal. The resulting new fuels are calledHyGasoline, Hy-Diesel and Hy-Coal. Since Hydrogen in general, and magnetically polarized Hydrogen in particular, have the highest known flame temperature among gaseous fuels (see the certification in www.magnegas.com), it is evident that the conversion of fossil fuels into Hy-Fuels eliminates hydrocarbons, $\mathrm{CO}$ and other combustible contaminants in the exhaust, because they are burned by Hydrogen. The importance of Santilli magnecular bonds is evident because of the impossibility of creating the new Hy-Fuels via the conventional valence bond and because, in the absence of the magnecular bond, we would have an unstable, quickly separated "mixture" of Hydrogen and fossil fuels of no industrial or environmental relevance.
Needless to say, as it is typically the case for all basic advances, the experimental confirmations of Santillimagnecules presented in this paper creates a number of open problems of fundamental chemical character, analytic detection and industrial application.

On fundamental grounds $[4,21]$, let us recall that molecules do not break down into components during thermal collisions due to the strength of their valence bond. Consequently, the Avogadro number remains constant for one mole of a molecular gaseous fuel under the increase of its pressure and temperature up to combustion, as established beyond doubt.

The situation is substantially different for magnecular gaseous fuels because their clusters do break down into components following thermal collisions due to the weakness of their bond, as established by Santilli in the original memoir [1] and confirmed in this paper. As indicated, magnecules do recombine following collision break down, thus maintaining the same pressure at the same temperature. Hence, Santilli concludes that subject to experimental confirmation or denial, the Avogadro number of a mole of magnecular gaseous fuel is expected to remain constant under constant pressure and temperature [4.21].

However, as confirmed in this paper, the recombination of magnecular constituents following thermal collisions decreases with the increase of temperature (see, e.g., the comparison of Figs. 6 and 7). Consequently, following observation of the behavior of magnegas under variations of pressure and temperature, Santilli states that subject to

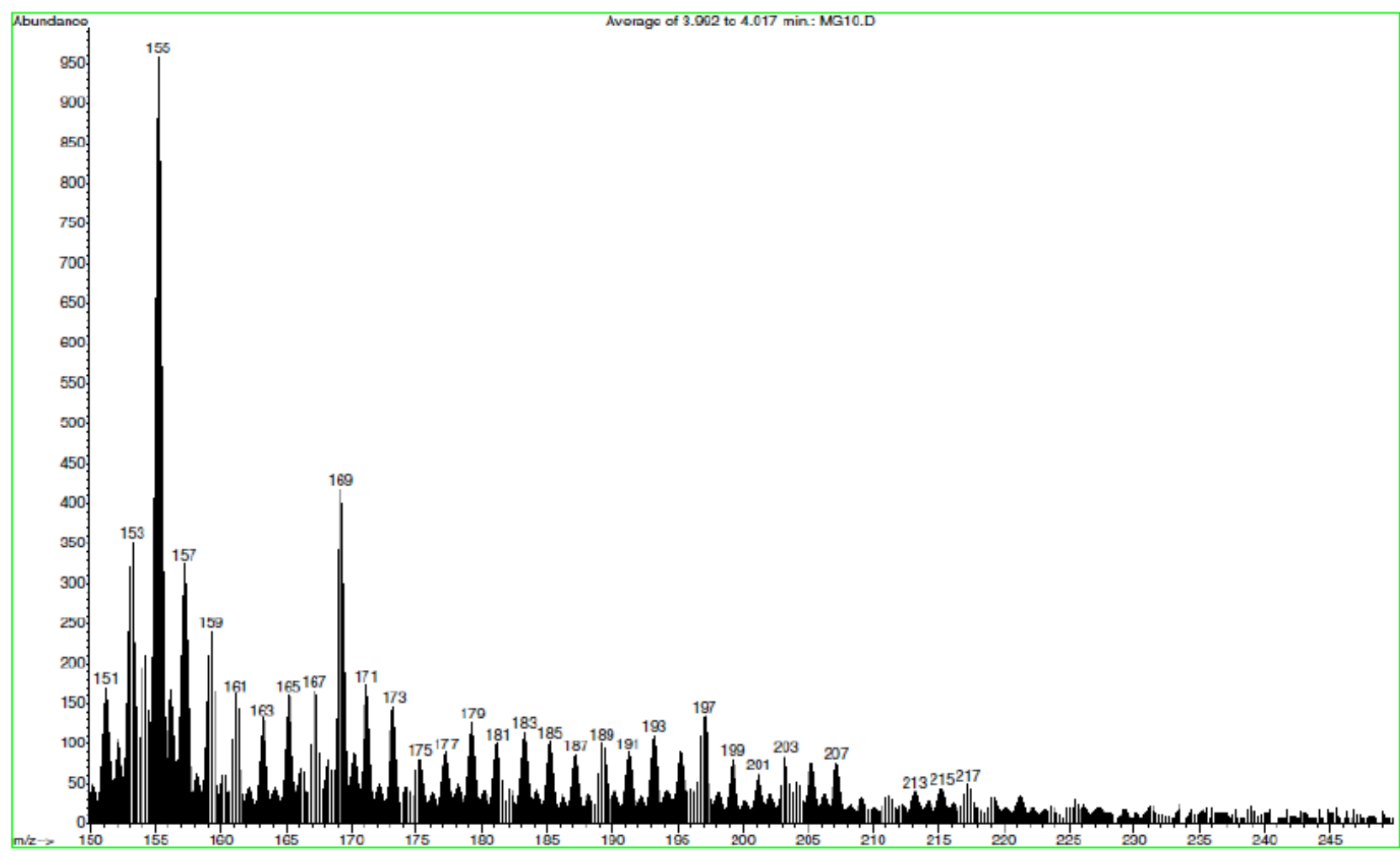

Fig. (18). One of numerous scans from the GC-MS operated at low temperature and with the long estpossible elusion time confirming Santilli's anomalous accretion by amagnecularcluster of one individual Hydrogen atom that can be observed from 2a.m.u. to hundreds of a.m.u. 


\begin{tabular}{|c|c|c|c|c|c|}
\hline $\begin{array}{l}\text { ORS REPORT NO. } \\
\text { DATE TESTED }\end{array}$ & $\begin{array}{c}185343-001 \\
4 / 10 / 2010\end{array}$ & & & & \\
\hline QUANTITY TESTED & 7 & & & & \\
\hline PACKAGE TYPE & standard & & & & \\
\hline SAMPLE & ID & A & B & 1 & 2 \\
\hline Mass & 2 & 1,531 & 3,343 & 288,163 & 185,549 \\
\hline Mass & 3 & 0 & 236 & 49,815 & 438,891 \\
\hline Mass & 4 & 0 & 203 & $12,648,080$ & $12,342,540$ \\
\hline Mass & 5 & 0 & 0 & 332 & 933 \\
\hline Mass & 6 & 0 & 0 & 13,260 & 12,020 \\
\hline Mass & 7 & 1,507 & 1,185 & 0 & 0 \\
\hline Mass & 8 & 380 & 0 & 0 & 0 \\
\hline Mass & 11 & 160 & 122 & 0 & 0 \\
\hline Mass & 12 & 619 & 158,821 & 4,850 & 9,025 \\
\hline Mass & 13 & 0 & 1,781 & 449 & 400 \\
\hline Mass & 14 & 887,617 & 745,201 & 57,902 & 11,191 \\
\hline Mass & 15 & 3,525 & 2,392 & 1,875 & 1,578 \\
\hline Mass & 16 & 286,293 & 259,183 & 24,627 & 16,952 \\
\hline Mass & 17 & 14,875 & 15,487 & 2,269 & 12,165 \\
\hline Mass & 18 & 50,806 & 52,423 & 10,248 & 104,140 \\
\hline Mass & 19 & 53 & 11,475 & 3,242 & 8,594 \\
\hline Mass & 20 & 12,301 & 11,149 & 8,302 & 71,458 \\
\hline Mass & 21 & 0 & 0 & 0 & 729 \\
\hline Mass & 22 & 0 & 19,942 & 222 & 159 \\
\hline Mass & 23 & 0 & 61 & 0 & 0 \\
\hline Mass & 24 & 0 & 0 & 182 & 218 \\
\hline Mass & 25 & 0 & 0 & 633 & 240 \\
\hline Mass & 26 & 220 & 180 & 2,838 & 1,408 \\
\hline Mass & 27 & 0 & 0 & 873 & 878 \\
\hline Mass & 28 & $8,188,280$ & $7,056,760$ & 536,530 & 125,200 \\
\hline Mass & 29 & 57,521 & 49,468 & 4,334 & 2,548 \\
\hline Mass & 30 & 10,389 & 8,393 & 3,618 & 5,306 \\
\hline Mass & 31 & 0 & 101 & 178 & 1,601 \\
\hline Mass & 32 & $1,880,953$ & 96,766 & 118,498 & 13,475 \\
\hline Mass & 33 & 1,339 & 0 & 139 & 483 \\
\hline Mass & 34 & 7,608 & 365 & 577 & 1,449 \\
\hline Mass & 35 & 0 & 0 & 0 & 225 \\
\hline Mass & 36 & 409 & 313 & 0 & 1,848 \\
\hline Mass & 37 & 0 & 0 & 0 & 79 \\
\hline Mass & 38 & 123 & 61 & 0 & 119 \\
\hline Mass & 39 & 301 & 0 & 308 & 433 \\
\hline Mass & 40 & 97,549 & 80,615 & 5,857 & 563 \\
\hline Mass & 41 & 463 & 81 & 209 & 328 \\
\hline Mass & 42 & 225 & 0 & 197 & 317 \\
\hline Mass & 43 & 480 & 0 & 113 & 295 \\
\hline Mass & 44 & 5,795 & $1,806,671$ & 14,262 & 13,828 \\
\hline Mass & 45 & 166 & 20,644 & 199 & 265 \\
\hline Mass & 46 & 60 & 7,265 & 98 & 159 \\
\hline Mass & 47 & 0 & 109 & 0 & 0 \\
\hline Mass & 48 & 0 & 0 & 0 & 0 \\
\hline
\end{tabular}

Fig. (19). Scan of various magnecular gases obtained by the Oneida Research Services with the equipment described in Sect. 2 providing additional independent confirmation that magnecular gases are indeed constituted by a series of detectable clusters detectable in the MS up to large a.m.u. (reduced in this figure to $48 \mathrm{a}^{\wedge} \mathrm{m}^{\wedge}$.u.), which clusters are however transparent to the IRD, as originally discovered by Santilli in $1998[1]$.

experimental confirmation or denial, the Avogadro number of one mole of a magnecular gaseous fuel is expected to increase with the increase of the temperature and decrease with the increase of the pressure $[4,21]$.

Since magnecular gaseous fuels are made available by the authors to qualified chemists at nocost (and, in any case, they can be purchase at very low costs in various countries), it is hoped that the chemical community resolves the sefundamental open issue sex perimentally, rather than via sole theoretical studies that, as such, can only present [personal opinions in view of the limited knowledge of this new field of chemistry at this time.

On grounds of analytic detection, recall that all currently available equipment and procedures have been specifically 
and solely developed for the detection of molecules. Therefore, we have a clear need of developing new gas chromatographers or other detection equipment and related procedures specifically intended for the detection of magnecules and their differentiation with molecules.

The best illustration of this need given by fact that, according to a large variety of analyses by qualified independent laboratories, depending on the selected liquid feedstock, MagneGas fuel contains from $30 \%$ to $35 \%$ Carbon Monoxide referred to the triple-bonded molecule $\mathrm{C}-\mathrm{O}$. Santilli calls these analyses "good faith experimental beliefs," in the sense that the instrument says so and that it's believed to be true without due scientific caution caused by the absence of independent chemical analysis on the same gas done with a different instrument.

In the event MagneGas did indeed contain $30 \%$ to $35 \%$ $\mathrm{CO}$, its exhaust should contain at least 25\% Carbon Dioxide $\mathrm{CO}_{2}$ (since $\mathrm{CO}$ is combustible). In reality, under proper combustion,independent certification of MagneGas combustion (see www.magnegas.com) has established the presence in said exhaust of about $5 \% \mathrm{CO}_{2}$, about $0.01 \% \mathrm{CO}$, up to $14 \%$ breathable Oxygen $\mathrm{O}_{2}$, no appreciable $\mathrm{HC}$ and $\mathrm{NO}_{\mathrm{x}}$ in ppm, the rest being given water vapor for (since MagneGas contains from $60 \%$ to $65 \%$ Hydrogen). It is evident that the sole presence in Magnegas exhaust of about $5 \% \mathrm{CO}_{2}$ and no appreciable combusted $\mathrm{CO}$ does indeed render the $30 \%$ to $35 \%$ CO in MagneGas a "belief."

The best representation of the contrasting data known to the authors is that provided by Refs. $[4,10]$. In essence, Santilli assumes that the chemical species detected by GC- MS in MagneGas at 28a.m.u. is composed partially by the conventional Carbon Monoxide $\mathrm{C}-\mathrm{O}$ and partially by the magnecular bond of the same atoms $\mathrm{C} \times \mathrm{O}$ essentially in the configuration of (Fig. 1). Santilli then argues that the high percentage of $\mathrm{CO}$ detected in MagneGas by various laboratories is in effect created by the equipment itself. Due to the known affinity between Carbon and Oxygen, the following reaction is in effect possible reaction $\mathrm{C} \times \mathrm{O}+$ trigger ${ }^{\wedge} \mathrm{CO}+$ heat, where the "trigger" is the detection mechanism of the instrument, and the release of heat is due to the fact that molecular bonds are much stronger than magnecular bonds.

Also, the high collisions occurring in combustion are expected to breakdown the weakly bonded magnecule $\mathrm{C} \times \mathrm{O}$ and produce the excess Oxygen needed to reach a quantitative understanding of the MagneGas exhaust.

The above scenario is so unknown at this writing that caution is suggested in predicting the percentage of $\mathrm{CO}$ existing in MagneGas from the measured percentage of $\mathrm{CO}_{2}$ in the exhaust (expectedly of about 7\%). In fact, available experimental data cannot exclude thepossibility that the entire content of the species in MagneGas with 28a.m.u. can be the magnecule $\mathrm{C} \times \mathrm{O}$, and the expected $7 \% \mathrm{CO}$ can in effect be created by collisions during combustion.

Needless to say, the authors are not in a position to ether deny or confirm such a reconciliation by Santilli between the atomic constituents of MagneGas and the molecular constituents of MagneGas exhaust, thus confirming the need for numerous additional measurements.

\section{CONCLUDING REMARKS}

As it is clearly set in scientific historians, the chemistry of the 18-th century was dominated by the belief that air, and gases in general, were composed of a mysterious substance called phlogiston. Only following one century of debates, Lavoisier, Avogadro, Cannizzaro and other illustrious scientists proved that phlogiston was a fictitious substance, and established the true molecular structure of air and other gases.

However, scientific history has the tendency of repeating itself. In fact, the widespread belief among contemporary chemists is that all composite structures throughout the universe, from the Hydrogen to the DNA, are "molecules," namely, clusters of atoms under one or another valence bond.

In the authors view, the historical significance of the chemical discoveries by the Italian- American physicist Ruggero Maria Santilli is to have established that, by no means, all atomic clusters in the universe can be reduced to molecules with a valence bond, because nature is immensely more complex than that.

Far from claiming any final understanding of the chemical structure of the universe, Santilli presented the following preliminary classification of atomic clusters with nonvalence bonds of increasing complexity [4,14]:

- IsoMagneCules (IMC), referred to clusters of the type studied in this paper under quantitative treatment by the single-valuedisochemistry;

- GenoMagneCules (GMC), referred to clusters requiring a quantitative treatment via the broader singlevaluedgenochemistry; and

- HyperMagneCules (HMC) referred to clusters whose complexity is beyond our understanding because it requires the more general, multi-valuedhyperchemistry particularly recommended by Santilli for deeper studies on the DNA.

All in all, it appears that, far from having achieved final knowledge of nature, structural advances in chemistry are indeed under way with so much yet to discovered and understood.

\section{CONFLICT OF INTEREST}

The author(s) confirm that this article content has no conflicts of interest.

\section{ACKNOWLEDGEMENT}

The authors would like to thank R. M. Santilli for countless consultations, repeated critical readings of this manuscript and in depth suggestions, without which this paper would not have seen the light of the day. Special thanks are due to the referees of the Open Physical Chemistry Journal for penetrating criticisms that have been crucial to achieve a better maturity of presentation. Additional thanks are due to all participants of the Seminar Course in Hadronic Mathematics,. Mechanics and Chemistry held on the Island of Kos, Greece, from September 19 to25, 2012. 


\section{NOTE ADDED}

Following the completion of this paper, various colleagues informed the authors of the recent paper.

K. K. Lange, E. I. Tellgren, M. R. Hoffmann, and T. Helgaker, "A Paramagnetic Bonding Mechanism for Diatomics in Strong Magnetic Fields," Science 337, 327 (2012).

Which presents a chemical species with a magnetic nonvalence bond predicted to exist under strong magnetic fields in certain astrophysical conditions. As one can see from the presentation in this paper and the quoted references, this species is a particular case of Santilli's magnecular bond, since the latter was conceived and tested as the most general possible configuration of electric and magnetic fields between two or more atoms with magnetically polarized orbitals suitable to produce an attraction. In fact, Santilli magnecular bond is also called "axial" or "perpendicular" in the sense indicated in (Fig. 1). In any case, the measurements presented in this paper constitute experimental evidence obtained on Earth that the magnetic bonds claimed by Lange et al. do indeed exist in astrophysics.

\section{REFERENCES}

[1] Santilli , R.M. Theoretical prediction and experimental verification of the new chemical species of magnecules. Hadronic J., 1998, 21, 789. Available at: http://www.santilli-foundation.org/docs/Santilli43.pdf

[2] Kucherenko, M. G.; Aringazin, A.K. Estimate of the polarized magnetic moment of the isoelectronium in the hydrogen molecule. Hadronic $J$., 1998 , 21, 895. Available at: http://www.santillifoundation.org/docs/estimate.pdf

[3] Aringazin, A .K. Toroidal configuration of the orbit of the electron of the hydrogen atom under strong external magnetic fields. Hadronic J., $\mathbf{2 0 0 1}, 24,134$. Available at: http://www.santillifoundation.org/docs/landau.pdf

[4] Santilli, R.M. Foundations of Hadronic Chemistry with Applications to New Clean Energies and Fuels. Kluwer Academic Publishers, Netherlands, 2001. Available at: http: //www.santillifoundation.org/docs/Santilli-113.pdf.

[5] Santilli, R. M. The novel magnecular species of hydrogen and oxygen with increased specific weight and energy content. Int. J. Hydrogen Energy, 2003, 28, 177-196. Available at: http://www.santilli-foundation.org/docs/Santilli-38.pdf

[6] Santilli, R. M. A new gaseous and combustible form of water. Int . J. Hydrogen Energy, 2006, 31, 1113. Available at: http://www.santilli-foundation.org/docs/Santilli-138.pdf

[7] Cloonan, M.O. Application of the Cplex-isoelectronic theory to electrocyclisations, sigmatropic rearrangements, cheletropic reactions and antiaromaticity: Consistent with Santilli'shadronic chemistry. Int. J. Hydrogen Energy, 2006, 32, 3026. Available at: http://www.santilli-foundation.org/docs/Cloonan-Paper2.pdf
[8] Cloonan, M.O. A new electronic theory of pericyclic chemistry and aromaticity is proposed: The Cplex-isoelectronic theory: Consistent with Santilli'shadronic chemistry, Int J. Hydrogen Energy, 2007, 32, 159. Available at: http://www.santillifoundation.org/docs/Cloonan-Paper1.pdf

[9] Cloonan, M.O. Origin of the endoselectivity observed in pericyciclic reactions based on the CPlex-Isoelectronic theory. Hadronic J., 2009, 32, 125. Available at: http://www.santillifoundation.org/docs/Cloonan-Paper3.pdf

[10] Santilli, R.M. The New Fuels with Magnecular Structure. International Academic Press, USA, 2008. Available at: http://www.i-br.org/docs/Fuels-Magnecular-StructureF.pdf, Italian translation available from the link: http://www.i-b-r.org/docs/Carb-StruttMagnecolare.doc

[11] Santilli, R.M. Experimental Confirmation of Nitrogen Synthesis from deuterium and Carbon without harmful radiations. New Adv. Phys., 2011, 4, 29. Available at: http://www.santillifoundation.org/docs/ICNF-1.pdf

[12] Brenna, R.; Kuliczkowski, T.; Ying, L. Verification of Santilli intermediate Controlled Nuclear Fusions without harmful radiations and the production of magnecular clusters. New Adv. Phys., 2011, 5, 9. Available at: http://www.santilli-foundation.org/docs/ICNF2.pdf

[13] Santilli, R.M. Additional Confirmation of the Intermediate Controlled Nuclear Fusions without harmful radiation or waste. In: Proceedings of the Third International Conference on the LieAdmissible Treatment of Irreversible Processes. Corda, C. Ed, Kathmandu University, 2011, pp.163-177. Available at: http://www.santilli-foundation.org/docs/ICNF-3.pdf

[14] Santilli, R.M. Isotopic, Genotopic and Hyperstructural Methods in Theoretical Biology. Naukova Dumka, Ukraine Academy of Science, Ukraine, 1997, Available at: http://www.santillifoundation.org/docs/santilli-67.pdf

[15] Yang, Y.; Kadeisvili, J. V.; Marton, S. Experimental Confirmations of Santilli's Magne Hydrogen. Int. J.Hydrogen Energy, 2013, 38, 5002. Available at: http://www.santillifoundation.org/docs/MagneHydrogen-2012.pdf

[16] Trell , E. Review of Santilli's Foundations of Hadronic Chemistry.Int. J. Hydrogen Energy.2003, 28, 251. Available at: http://www.santilli-foundation.org/docs/Trell-review-HC.pdf

[17] Tandge, V. Hadronic Chemistry Applied to Hydrogen and Water Molecules. AIP Conf. Proc., 2012, 1479, 1013. Available at: http://www.santilli-foundation.org/docs/Tandge-AIP.pdf

[18] Gandzha, I .; Kadeisvili , J. New Sciences for a New Era: Mathematical, Physical and Chemical Discoveries of Ruggero Maria Santilli, Sankata Printing Press, Nepal, 2011. Available at: http://www.santilli-foundation.org/docs/RMS.pdf

[19] Santilli, R .M . Apparent detection of $\mathrm{H} 3 \mathrm{O}, \mathrm{COH}, \mathrm{CO} 2 \mathrm{H}$ and other anomalous species. [submitted for publication], Available at: http://www.santilli-foundation.org/docs/H3O-paper.pdf

[20] Santilli , R.M . A study on the new class of Hy-Fuelss without toxic contaminants in their exhaust.preprint IBR-CH-03-13, 2013, [Epub ahead of print]. See also Santilli's recent international patent applications and the webpage of Magnegas Corporation Available at: http://magnegas.com/hy-fuels

[21] Santilli, R.M. Experimental proposal to confirm or deny the variation of Avogadro number for magnecular gases.preprint IBR-CH13-09, 2013, [to appear].

(C) Yang et al.; Licensee Bentham Open.

This is an open access article licensed under the terms of the Creative Commons Attribution Non-Commercial License (http://creativecommons.org/licenses/by-nc/3.0/) which permits unrestricted, non-commercial use, distribution and reproduction in any medium, provided the work is properly cited. 